\title{
Au cœur du sujet : le corps en objets?
}

Jean-Luc Jamard

\section{OpenEdition}

Journals

Édition électronique

URL : https://journals.openedition.org/tc/197

DOI : $10.4000 /$ tc. 197

ISSN : 1952-420X

\section{Éditeur}

Éditions de l'EHESS

\section{Édition imprimée}

Date de publication : 1 juin 2002

ISSN : 0248-6016

\section{Référence électronique}

Jean-Luc Jamard, «Au cœur du sujet : le corps en objets ? », Techniques \& Culture [En ligne], 39 | 2002, mis en ligne le 13 juin 2006, consulté le 29 septembre 2022. URL : http://journals.openedition.org/tc/ 197 ; DOI : https://doi.org/10.4000/tc.197

Ce document a été généré automatiquement le 29 septembre 2022

Tous droits réservés 


\title{
Au cœur du sujet : le corps en objets?
}

\author{
Jean-Luc Jamard
}

1 Édifier «une théorie de la culture matérielle à partir de ce qui lui est essentiel : la matérialité dans son rapport aux conduites motrices du sujet, comme matrice de subjectivation", voilà l'objectif que Jean-Pierre Warnier assigne (pp. 16-17) à son dernier livre en date, publié en 1999 (ci-après CC) ${ }^{1}$. Le sous-titre de l'ouvrage, lui aussi, donne le ton : L'homme qui pensait avec ses doigts -l'expression est empruntée à Marcel Mauss citant Maurice Halbwachs ${ }^{2}$. Au fil de ses pages, revient l'idée de «mise en objets » que définit, en bref, l'incorporation, par le sujet, non des choses elles-mêmes puisqu'il ne s'agit pas de prothèses au sens strict- mais de leur dynamique, et qui traduit l'anglais objectification (cf. Miller 1987). Ailleurs, dans un recueil collectif (AM) ${ }^{3}$ paru, en 1999 également, sous la direction de Marie-Pierre Julien et de notre auteur, celui-ci en appelle à une "anthropologie phénoménologique» qui cesserait «de ricocher sur la matière et de rebondir vers le ciel des repré-sentations » pour se nourrir -enfin !- d'une " ethnographie des conduites-motrices-dans-les-objets », en vue « de faire la théorie du rapport sujet-objet-autre sujet investi de déterminations multiples » (AM : 140).

2 Conduites motrices, mise en objets... Techniques du corps et au-delà, ou en amont ? Du moins aura-t-on compris que J.-P. Warnier, comme ses partenaires du groupe de recherche MàP ("Matière à Penser", bien loin de la matière pensée par Changeux \& Connes 1989), qu'il a promu à l'Université René-Descartes, recueille chez Mauss nombre de fruits. Mais il veut en tirer un vin nouveau grâce aux « techniques de soi ", aux objets « subjectivants " et même aux « gouvernementalités », toutes notions où se discerne l'influence foucaldienne du reste ouvertement revendiquée au sein du collectif MàP.

3 On prêtera l'oreille, j'espère, si je le montre comptant sur les développements du programme maussien pour réduire "l'aporie de la causalité réciproque des faits techniques et des faits de société... "dichotomie fatale" [écrivent Latour et Lemonnier (1994)]... », car " partir des sujets agissants par les conduites motrices dans un monde 
d'objets permettrait [...] de progresser dans l'analyse de ce que Latour (1991) appelle un "hybride socio-technique" " (CC: 166). Et pour fixer davantage l'attention de nos lecteurs, j'ajouterai que, à l'entendre encore, la tradition savante dont il se sent «le plus proche est celle de la technologie culturelle, développée [...] par A. Leroi-Gourhan [...], A.-G. Haudricourt [...], R. Cresswell (cf. la revue Techniques \& culture), et les membres du laboratoire "Techniques et culture" »; une tradition néanmoins à reprendre afin de la "déborder largement ", elle et sa "préoccupation lancinante pour l'action efficace sur la matière » (CC:16, ital. dans le texte).

Généalogies : la tête et les jambes

Quelle est au juste la thérapie possible de la douloureuse obsession ci-devant déplorée ? Nous y viendrons. Afin de déboucher sur ses propres vues, Warnier retrace au préalable les grandes lignes de "trois paradigmes ", parmi les dispositifs d'explication qui, depuis Darwin, se succédèrent à l'avant-scène des sciences du passé pour rendre compte du processus d'hominisation: le "cérébraliste », le «bipédique», enfin le "culturobipédique » (CC, chap. 2).

5 Cérébralisme: dans le phylum où l'on peut situer les ancêtres de notre espèce, la sélection naturelle aurait d'abord primé des changements qui amé-liorent certaines performances du cerveau, moyennant le développement de son cortex et du néocortex. D'abord la grosse tête, puis les jambes et le corps suivent ${ }^{4} . .$. Et surtout, les capacités nouvelles de l'intelligence apparaissent sous cet angle comme la cause proximale de l'émergence d'une culture matérielle.

6 Mais après que Raymond Dart eut mis au jour le premier australopithèque en 1924, l'«enfant de Taung», la perspective se renverse à terme, malgré les résistances durables de la plupart des paléo-anthropologues, dont, notons-le au passage, les présupposés cérébralistes, déjà mis à mal par le célèbre Pithecanthropus erectus de Java (1891), avaient pourtant tenu bon jusqu'à la Seconde Guerre mondiale. C'est ainsi que le paradigme bipédique prend son essor, car «Il fallait se rendre à l'humble évidence : [l'hominisation] avait commencé par les pieds» (CC: 39). Warnier résume deux variantes d'une telle conception, celle d'André Leroi-Gourhan, "téléologique et adarwinienne »-des réflexions de Nathan Schlanger (1994) sont évoquées à l'appui de ces qualificatifs-, puis celle de Sherwood L. Washburn, "darwinienne et non finaliste ». Cependant, si les écrits de Leroi-Gourhan dans leur ensemble, peu explicites sur ce point, dénotent sans conteste certaines influences philosophiques bergsoniennes (la tendance progressiste dans la durée; l'« évolution créatrice»?), voire teilhardiennes, je ne crois pas qu'en matière d'hominisation envisagée sur le plan anatomique, celles-ci aient trop infléchi méthodes, raisonnements et conclusions. En bref :

« [la] vision "cérébrale" de l'évolution paraît maintenant inexacte et il semble que la documentation soit suffisante pour démontrer que le cerveau a profité de l'adaptation locomotrice, au lieu de la provoquer. (Leroi-Gourhan 1964: 42, cité dans $C C: 40)$.

7 Thèse que rien ne saurait encore réfuter aujourd'hui. À vrai dire, dans les domaines connexes, certaines données disponibles pour le grand ethnologue-préhistorien, sous notre regard rétrospectif, peuvent forcément sembler incomplètes ou périmées ou, disons, soustraites à son interprétation en raison de quelques partis pris, malgré un cadre théorique qui tendrait à les intégrer; ainsi François Sigaut (1990 : 34) remarquaitil que «Leroi-Gourhan ne se pose aucune question sur l'emploi de percuteurs chez les primates [non humains], ce qui est réellement surprenant». Les travaux des 
primatologues, justement, nous ramènent au chapitre commenté. La seconde variante du "bipédisme " y est illustrée par ceux de S. L. Washburn (1965) qui, conformément à l'orthodoxie néodarwinienne dont ils se réclamaient, connurent après leur entrée en lice la fortune puis la transformation, sinon l'extinction, face à la concurrence de faits et débats inédits... De quoi s'agit-il, en très gros ? Par suite de changements climatiques, à la forêt est-africaine, se serait substituée une savane arborée où des hominoïdes étaient contraints de "se redresser» lors des déplacements entre les massifs d'arbres. D'où, au fil du temps, la sélection par reproduction différentielle, moyennant mutations aléatoires, des individus les mieux doués pour cette nouvelle locomotion triplement avantageuse: meilleur repérage des prédateurs dans les hautes herbes, posture d'intimidation à leur encontre, aptitude, aussi, au transport d'objets.

8 Sur ce, Warnier fait, des acquis de la primatologie dans l'après-guerre et surtout ces dernières années, la ruine du "scénario Washburn", lequel en avait tout d'abord bénéficié. De là son insistance envers le dernier cri, le "para-digme culturobipédique $»^{5}$, comme la mienne quant à la généalogie qu'il pro-pose, décisive selon moi pour son vaste dessein, car elle inscrit ce dernier dans la profondeur d'une genèse et du genre humain et de ses paléontologies.

Déjà quelque peu "cultivés", manieurs d'outils mais pas vraiment bipèdes, tels sont devenus, à nos yeux dessillés, certains primates non humains, nul n'est censé l'ignorer parmi nous (outre, depuis les années cinquante, un nombre grandissant de publications toujours plus popularisées, cf. Cresswell et al. 1994, discuté dans CC: 45-46). Apprentissages, traditions techniques distinctes au sein d'une même espèce, organisations sociales et jusqu'aux "politiques» simiennes, sont introduits dans nombre d'interprétations de la primatologie comparée. Et cela, écrit notre auteur, "bien avant l'acquisition de la bipédie, de sorte que celle-ci n'est pas forcément due, au point de départ, à une mutation génétique sélectionnée par la pression environnementale, mais à une innovation comportementale, voire "culturelle" validée après coup par des boucles de rétroaction de type darwinien » ( $C C: 43$, je souligne). Sauf erreur néanmoins, les paléontologues penchent de nos jours pour une intelligence de la bipédie permanente -inaugurée chez nos lointains ancêtres ou cousins disparus- en termes d'exaptation antérieure de quelques espèces arboricoles dont les prédispositions (et expériences) bipèdes seraient l'effet secondaire, indirect, le corrélat neutre d'un ajustement directement sélectif à leur mode de vie propre. Autrement dit, d'une préadaptation, le "pré-» comme d'ailleurs le bien avant souligné plus haut (bonobos, chimpanzés..., seuls observables in vivo: «bien avant » quoi ?) ne pouvant s'admettre que faute de mieux, en flash-back explicite - sinon, gare au finalisme ou au retour de l'orthogenèse débridée, que pourtant Warnier discrédite avec raison. Et la "validation » des innovations comportementales à comprendre dans le strict cadre de la biologie évolutionniste... problème redoutable. Restons-en là. Car mon détour conduit déjà à la question capitale.

10 "L'hominisation par la matière », formulation (et titre du chapitre 2) établie sur tous les rappels résumés jusque-là, constitue comme une roche-mère de toute la réflexion présentée ici, bien que les différentes références soient légion qui vont ensuite s'appliquer à l'analyse des faits les plus actuels.

«La trajectoire générale de l'hominisation commencerait avec le choix comportemental [de la bipédie], qui donne une prime sélective à la refonctionnalisation des mains et leur spécialisation dans la manipulation ludique et pratique des matières et de l'outillage. » (CC: 47); « La locomotion bipède fait partie 
d'une stratégie comportementale. [Les] changements affectant le comportement ont toute chance de précéder les changements anatomiques de première grandeur, et l'on peut s'attendre à ce que le comportement procure les conditions de la sélection en fonction desquelles les nouvelles configurations anatomiques ont la possibilité d'évoluer.» (Foley 1995: 136, cité dans CC: 51, trad. Warnier, mes italiques). "L'essentiel du "travail" des primates et des hominiens au cours de l'évolution, aura sans doute été de produire du social en agissant sur soi et les uns sur les autres, en élaborant des conduites motrices appuyées sur les objets, plutôt que de produire des moyens de subsistance en instrumentalisant des objets dont la destination ultime aurait été de devenir des outils. » ( $C C: 46)$.

11 À ces phrases-clés s'ajoute un constat général qui ne saurait être faux. Du paradigme culturo-bipédique, se déduit la médiation des «multiples relations entre le biologique, le social, le culturel » par « les conduites motrices et la matière que le corps met en mouvement » (CC: 51$)$. Mais voici l'idée-force, selon laquelle « Le rapport privilégié de l'homme à la matière affecte tous les aspects de l'être-homme : le jeu, le développement de l'enfant, l'inconscient, la socialité, l'affectivité, le corps, l'art. L'outillage n'est qu'un cas parmi d'autres qu'une civilisation moins entichée de technique que la nôtre mettrait à sa juste place: pas la première». Ipso facto, comme Mauss «en a eu l'intuition, la dynamique des objets matériels incorporés aux conduites motrices se trouve être en relation nécessaire » avec tout l'humain ( $C C: 58)$. Dont acte.

Incarnations

12 Tout l'humain : y compris, on vient de le voir, Homo ludens. Et que faber baisse un peu le ton (gardons cela en mémoire). Précisément, dans l'autre ouvrage commenté, Pierre Parlebas traite des jeux sportifs ${ }^{6}$ et conflue avec le précédent quand il pose que « La technique du corps incorpore les objets ma-tériels. Une raquette de tennis, les roues de la bicyclette, la proue du bateau ou les spatules des skis vont prolonger le corps et devenir des capteurs sensoriels. [...] Les objets matériels sont partie prenante de la pratique corporelle » (AM : 37; quoi de plus merleau-pontien...). Mauss n'aurait pas su le voir, malgré «l'aspect novateur de ses positions ». (Warnier se chagrine lui aussi de ce que chez l'auteur des "Techniques du corps ", au demeurant père fondateur avéré, on constate un bougé du départ entre celles-ci et les techniques instrumentales; il en serait resté par le fait même à la seule "intuition » touchant l'incorporation de la dynamique des objets; $c f$. ci-dessus et $C C$ : chap. 1, passim.) P. Parlebas, pour sa part, présente un cadre d'analyse fort original des techniques sportives qui contraste avec la réflexion et l'enseignement établis dans le domaine de l'éducation physique, où se juxtaposent des savoirs disparates sur le corps, tirés de la physiologie, la biomécanique, l'ergonomie, la psychologie, la sociologie... À ses propres connaissances comme à son expérience du sujet, il entend intégrer deux apports irrécusables de Mauss -le relief donné aux déterminants socioculturels des techniques du corps et la conception de l'« homme total »-, mais en franchissant certaines barrières qui les limitaient. De la sorte, il définit une terminologie et une taxinomie par quoi se révèle enfin comment, dans les jeux sportifs, « la culture matérielle [...] pénètre les techniques du corps » (AM : 39). Comment, aussi, tant l'espace que la logique interne du jeu modèlent les agents et leurs actions, réciproques ou non. Si bien que dans une perspective résolument praxéologique ( J'agis, donc je suis », AM:34), Parlebas réactive les catégories frappées de son estampille : fait praxique total, ludomotricité, sémiomotricité, psychomotricité, ethnomotricité, sociomotricité, éco-jeux, techno-jeux... Quels sont les contenus et l'efficience de ces innovations conceptuelles? Le lecteur mis en appétit se plaira à le découvrir sur pièces ${ }^{7}$. 
Que la dynamique de l'objet s'incarne dans le corps en jeu, si l'on peut dire, c'est à mon sens indéniable. Même quand l'artefact n'est plus là qu'à l'état de traces mémorielles, d'engrammes discernables dans les "conduites motrices». Par exemple, plusieurs mouvements d'esquive et de projection en aïkido semblent témoigner de la présence d'un fantôme de sabre ou d'un sabre virtuel, rémanence d'une ancienne technique d'objet dans la technique du corps. Et le judo traditionnel inclut certains kata ("formes ", exercices formels) qui évoquent, explicitement cette fois, des corps à corps en armure. À l'inverse, dans des entraînements à la fois "joués" et "dansés " au combat, les armes sont seulement figurées par les gestes qui anticipent leur maniement : nombre de cas ethnographiques, et surtout la pyrrhique de la Grèce antique, gymnopédie initiatique incorporant chez les jeunes gens l'action de la panoplie, sont bien connus sur ce point. En fait, plus d'un ethnologue a pensé de longue date que les techniques du corps "deviennent une mémoire» quasi incons-ciente, "tenace et durable » (Kawada $1990: 111)$.

«Franz Boas (1927) [...] a remarqué que la formation et la transmission du "style" en art primitif sont assurées par des "motor habits" [... proches des techniques du corps de Mauss...], mémoire corporelle survivant quelquefois aux changements matériels superficiels et formant un des aspects de la couche profonde et inconsciente de la "longue durée".» (ibid. : 124).

Sur ce fond, pourrait s'inscrire la fine et sensible contribution de Céline Rosselin ${ }^{8}$. Mais elle concerne des processus à court terme, l'incorporation et la "désincorporation " d'ustensiles d'un tout autre genre, les objets et le mobilier répartis dans les logements " une-pièce ", repérés lors des changements de domicile. L'auteur y insiste: il s'agit bien de processus liés «à une mémorisation corporelle inconsciente» (AM: 110); leur compréhension ne saurait s'arrêter aux constats des sujets, forcément suscités a posteriori par la demande expresse de l'ethnologue, lequel, du coup, doit privilégier les gestes, palliatifs des lacunes du discours ou indices d'embarras -imputables aux habitudes antérieurement acquises- dans l'« apprentissage " progressif de nouveaux espaces privés. Pour ce faire, C. Rosselin en appelle aux concepts de "schéma corporel » et d' ' image du corps », comme d'ailleurs la plupart des chercheurs dont il est question ici (qui renvoient là-dessus à Paul Schilder [1980] et Maurice MerleauPonty [1997], entre autres références moins connues). Résultat de ses enquêtes :

« Si l'incorporation conduit à un rapport évident, allant de soi, avec les objets et l'espace, cette évidence est une construction qui se réalise dans la confrontation avec la matière, souvent avec hésitations et maladresses, toujours par expérimentation. [...] L'espace à habiter et ses objets sont incorporés à partir du moment où les habitants ne peuvent pas en décrire le processus. » (AM : 111).

15 À propos d'un «terrain » analogue et dans la même veine, Tim Putnam et Valérie Swales analysent certains effets désorganisateurs du déménagement sur les « routines» incorporées de la «fabrication d'un chez-soi » (home en v.o., je présume) ${ }^{9}$. Sous cet angle encore, emménager perturbe les conduites motrices établies. C'est le moment crucial, selon ce texte aussi, d'une remontée vers la conscience de schèmes qui demeurent d'ordinaire «inconscients». Mais il s'ensuit un inconfort parfois douloureux altérant jusqu'à l'identité du sujet (au sens fort). Car celui-ci se «met en objets " dans son home et se construit quotidiennement en agissant sur lui-même au travers de ses gestes, donc de son rapport aux choses. Qu'il y ait changement, et voilà le trouble introduit, avant " reconstruction » de tout le dispositif incluant l'être humain et son environnement domestique. Les deux auteurs -cuisine et salle d'eau sont 
privilégiées par leurs observations- discutent une bibliographie abondante (en l'espèce, $c f$. un livre français déjà classique : Kaufmann 1997).

Des agencements de gestes banals; et non conscients... ou presque : un passage de LeroiGourhan (1965, section "Les chaînes opératoires machinales » : 26-31), bref mais fort parlant, s'y réfère en les démarquant soigneusement de purs "automatismes » biologiques.

«[...] la plupart des chaînes que nous déroulons du réveil au coucher n'appellent qu'une faible intervention consciente; elles se dévident non pas dans l'automatisme pour lequel l'intervention de la conscience serait nulle, mais dans une pénombre psychique dont le sujet ne sort qu'en cas d'imprévu dans le déroulement des séquences. Dans les gestes qu'on enchaîne au cours de la toilette, du repas, de l'écriture, des déplacements et transports, si exceptionnel que soit le retour à la lucidité, il est décisif; c'est pourquoi le terme de "chaînes opératoires machinales" et non automatiques, inconscientes ou instinctives, me paraît devoir être appliqué.» (LeroiGourhan 1965: 29, mes italiques; voir le contexte, où ces "chaînes» sont rapportées à l'apprentissage précoce dans ses variantes sociales et culturelles, sans pour autant que Mauss soit cité).

Bien sûr, le mot "sujet", sous cette plume concise, n'a pas les acceptions phénoménologique, métapsychologique ou foucaldienne tant prisées par Warnier et quelques collègues du MàP. D'aucuns, peut-être emportés par l'attraction sémantique qu'elles exercent, plaident pour une « anthropologie du singulier » (e.g. AM:117) qui ne va pas de soi : oxymore délibéré ? Et plusieurs penseurs de l'homme en société sont appelés à la rescousse. Moi qui croyais contribuer, par profession, à la comparaison de variétés d'humanité, dans l'espace et le temps, y compris pour déceler un beau jour l'unité sous la diversité ou vice versa... Aux temps du Christ, devant la foule des disciples, un bon pasteur prêche: "Chacun de vous, humains, est un être à part, différent de tout autre... -Sauf moi! » coupe un fidèle ${ }^{10}$. Jolie mise scène d'un vieux problème pour l'Occident. L'individuel est indicible, clamaient certains scolastiques (n'était mon autocensure, je retomberais dans la querelle des universaux). Foin des disputes de catégories dans le ciel des idées. Suivant les cadres de références et les incidences d'une recherche empirique, l'individué sera le sujet, ou l'agent, « rationnel » ou non, le «collectif» latourien, ou bien l'organisme, voire un réseau de représentations circulant entre cerveaux (pour tel cognitiviste pur et dur). Ou encore l'«acteur social». Mais la société justement, le groupe ethnique, la culture, le fait technique, le fait social irréductible, le système de rapports sociaux? À chacun ses singuliers.

18 Toutefois, n'allez pas croire que les écrits en question ici font litière de ces derniers cadrages. Non, certains y marient ouvertement, dans l'application, leurs anthropologies du sujet (outre ceux, nous l'avons vu ou le verrons, de Parlebas et de Warnier). Ainsi en est-il des pages très neuves, à ma connaissance, pour l'ethnographie du proche au présent, qu'Agnès Jeanjean consacre au traitement des déchets et des eaux usées dans les égouts de Montpellier ${ }^{11}$. Elles s'attachent à "l'action de percevoir et à ses conséquences sur la construction individuelle et le positionnement social des sujets qui perçoivent» (Marie-Pierre Julien, AM «Introduction»: 24). Attendu que «Les dispositifs techniques, leur histoire et les gestes qu'ils impliquent renvoient bien évidemment à des rapports sociaux » (Jeanjean, AM : 73), deux groupes d'ouvriers sont mis en perspective : l'un réunit les " égoutiers " municipaux, l'autre étant également chargé de l'assainissement mais employé, lui, par une entreprise privée. D’après les premiers, toucher l'excrémentiel, surtout à mains nues, et le flairer, conditionne 
l'expertise opératoire, prend figure de transgression, effraie le public (pour leur grande fierté), les valorise parce qu'ils sont en contact avec des substances dangereuses à tous égards, y compris symboliquement. Mais «fortifiantes » pour eux! En revanche, ceux qui relèvent du privé ne se targuent nullement d'un savoir par les sens, ni d'un surcroît de forces tiré de l'immondice. Bien au contraire: ils craignent la contagion par contiguïté, s'en protègent (peur du Sida, notamment), et rationalisent leurs compétences. Autre différence, donc, "les égoutiers [municipaux] effacent la technique " et l'outil (AM: 80), alors que leurs homologues du marché libre la revendiquent... Au bout du compte, tous les contrastes sont rapportés à l'ancrage des uns ou la mobilité des autres dans un système local de relations d'agents avec les institutions, et à l'organisation du travail; celle-ci dépend des statuts socioprofessionnels des groupes comparés, « de l'idéologie et des valeurs qui la portent [...], des rapports sociaux qu'elle sécrète et dont elle est en même temps l'expression » (AM : 84).

Voilà qui, par plusieurs entrées, nous rapproche de trois chapitres de ce même ouvrage, AM.

D'abord, Frédérique Lecœur, «nez » chez un parfumeur, évoque l'articulation olfactive des sensations et des représentations, sur laquelle se fonde son métier, lors d'un entretien que mène Céline Rosselin ${ }^{12}$. «Le professionnel... » [résume M.-P. Julien dans son "Introduction»], utilise à la fois sa mémoire personnelle, impliquant ses sensations et sa mémoire individuelle, et son appartenance à la société » (AM : 25). En ce domaine, les percepts doivent souvent être exprimés dans des registres autres que celui de l'odorat. Ils indexent le souvenir d'émotions jadis incorporées (tiens, comme les rêves le font par médiations pour la neurobiologie ou, autrement, pour la psychanalyse, non ?); et chaque personne s'est construit son propre référentiel mais toute culture a son univers, avec ses codages, avec ses valeurs ( $c f$. F. Lecœur, AM: 90, 93-94).

21 En deuxième lieu, le texte de François Hoarau circonscrit le « rapport au déchet ». Là cependant, point d'égoutiers, c'est de récupération et de tri d'objets usagés qu'il s'agit ${ }^{13}$. Le rebut n'est pas du seul ressort d'une taxinomie sur la gamme symbolique, «...il est également produit par l'action de "jeter" " (AM : 97-98, mes italiques). L'acte même du trieur, observé et pratiqué par l'auteur au sein de la Communauté d'Emmaüs à Besançon, et que soient triés des assiettes, du linge, des livres..., "crée " une marchandise de prix ou un déchet. L'un de ces statuts et les attributs d'entre-deux, l'objet les tiendra du geste par lequel on le lance sans ménagement ou on l'entrepose avec soin. La simple estimation par le regard n'y suffit pas. L'apprentissage du savoirfaire indispensable met en jeu toucher, odorat, motricité (que structure la matière, par ailleurs « subjectivante » car il n'y a pas de standards gestuels), tentatives d'imitation des Compagnons... Et représentations internes : sur ce point, F. Hoarau s'essaie -c'est son originalité- à reconstruire ses faits grâce au modèle de l'action qu'a élaboré le spécialiste des neurosciences Marc Jeannerod, où l'imagerie motrice joue un rôle capital. La représentation $d u$ but pilote l'action (plus que la réactivité directe envers «le monde ») ${ }^{14}$. Par suite, «..."l'observation des mouvements d'autrui n'est pas simplement [celle] d'un pattern visuel, mais la génération d'une image motrice de soi-même accomplissant la même chose que l'acteur observé" " (AM : 104, citation d'une source qui expose le «modèle Jeannerod»). 

(lato sensu) dans une communauté d'agents et plus largement dans la cité: Nicolas Auray nous donne à voir des groupes de pirates de l'informatique (hackers), dont les pratiques engendrent une éthique propre, jointive d'un nouveau « civisme $»^{15}$. La collectivité qu'étudie N. Auray se consacre à la production de "démos" (alias « démonstrations »), de brèves animations audiovisuelles qui sont destinées à mettre en valeur les propriétés de tel ou tel micro-ordinateur. Mais cette finalité initiale est détournée. En effet, les membres du collectif ont acquis sur le tas un savoir de programmeurs (souvent issu d'une passion pour les jeux vidéo) qu'ils commencèrent à appliquer de façon hétérodoxe en composant des logiciels inédits à partir du "cassage " des protections de démos fournies par les marques. Or, ce faisant, leur activité prend un tour chaque fois plus civique, plus politique : conception de démos parodiques qui moquent les leaders du marché, dénigrement, en actes, de la formation institutionnelle, promotion de la liberté d'accès à l'information... Autant de brèches où vont s'engouffrer la critique radicale de certains monopoles aussi bien qu'une « réflexion citoyenne », stimulée par ce biais, « sur l'usage de l'informatique » (AM : 70).

Pareille « ethnographie des usages de l'ordinateur» (Auray), usages pensables comme «corps à corps avec la machine " (Julien, AM: 24), devra, je pense, rejoindre les investigations qui touchent aux techniques de l'intellect - par conséquent du corps et de la matière, est-ce vraiment surprenant... Et qu'on ne dise pas que si tout s'avère « technique » en droit, rien ne le reste en faits pertinents : c'est hors sujet. L'écriture, ses supports, où les situer? Influent-ils, oui ou non, sur les opérations de la pensée, de la mémoire, sur l'organisation sociale (cf.évidemment Goody 1979)? Quant à l'imprimerie, Elizabeth Eisenstein (1991) en fit ressortir le rôle, majeur dès sa diffusion (qui accrut copieusement la possibilité de confronter et, partant, de critiquer, les écrits ou autres documents, in extenso), dans la révolution scientifique coperniciennegaliléenne. Plus: dans la dynamique de la Renaissance. Notre "raison» est bien «graphique ». En résultent de profondes corroborations des effets causés par les procédés d'objectivation de la pensée verbalisée. En ira-t-il autrement de l'usage, si varié et si vite propagé, de l'informatique, y compris pour le plus simple des traitements de texte, où perdure la lettre ? Laissons cela, mais à n'en pas douter, auraisje couché sur le papier, au crayon -difficile, vu les habitudes prises-, le présent article, qu'il eût été différent (et meilleur ou pire). Tout court, ou «micro ", l'ordinateur ne «pense » pas mais «fait penser» d'une manière sui generis (cf. Selz 1994). À point nommé, empruntons le langage du MàP et de Michel Foucault, en réciprocité de perspectives, et nous voilà "subjectivés ", chacun pour soi, par le maniement de nos computeurs, grâce aux faveurs d'une petite souris qui d'un geste, nous facilite l'incorporation de leurs propriétés matérielles et logiques, savoirs humains objectivés ${ }^{16}$.

Revenons à la morale, à l'éthique, mais aussi aux incorporations et aux gestuelles. Richard Pottier, connaisseur en éducation confucéenne dans l'enceinte familiale au Viêt-nam, démontre sur pièces que les discours, tant glosés par les ethnologues, restent cois devant quantité de significations ${ }^{17}$. Celles-là mêmes qui relèvent d'une culture «religieuse " sont souvent mieux déchiffrables dans certaines conduites corporelles retransmises depuis des lustres. (Peut-être P. Parlebas trouverait-il à exporter vers ce terrain -avec l'accord de l'autorité d'accueil, s'entend- la catégorie de « sémiomotricité ", i.e. inter-motricité signifiante, si je ne m'abuse.) La famille est le cadre où se reproduit l'ethos confucéen; en son sein, les rôles se révèlent normalisés, 
gouvernés par les principes : point de « rites » au sens courant pour le confucianisme, mais, traduits littéralement, des "normes» et "principes» (AM: 47). Or voici: l'hypothèse centrale qui étaie la contribution de R. Pottier est que « dans l'éducation confucéenne, le conditionnement corporel imposé aux enfants vise à obtenir, de leur part, une "incorporation" de la signification religieuse des relations intra-familiales " (AM : 48). Les gestes de la mère à l'égard du bébé, différenciés suivant son sexe, le signalent déjà, mais outre ces expressions de la tendresse, comme d'autres encore, entre conjoints, ou bien germains (aînés et cadets, de sexes identiques, opposés), et envers le père ou la mère, on le perçoit dans les multiples manières de saluer tout ce monde, effigies des ancêtres comprises. L'ensemble fait l'objet, de génération en génération, d'une mimesis reproductive façon Bourdieu, qui court-circuite la conscience du sujet en inscrivant une morale dans son mouvement, dans ses attitudes; d'où cette pérennité de l'ethos confucéen, confondante à première vue, puisque la lecture des textes fondateurs, bien qu'ils existent, fut toujours un apanage restrictif.

D'après Pottier, dans son exemple, le sens échoit au geste plus qu'à la parole et bien plus qu'à l'écrit; mais justement, "Le Ciel ne parle pas », disait Confucius (ou l'ai-je rêvé ?). En tout cas, si sa morale ressortit vraiment au grand genre " religion ", c'est de la religion incarnée.

Épiphanie

Une idéologie incarnée ${ }^{18}$ : c'est aussi de cela que traite Warnier, à mes yeux, dans "L'ethnographie de la mise en objets» ( $C C$, chap. 3); morceau de bravoure, ce me semble. Car de plus, il rend manifeste un réel imaginable et formant système qui, sans l'effort de l'herméneute étranger, demeurerait caché, n'ayant aucune expression vernaculaire graphique, ni discursive organisée, à la différence du confucianisme ou d'autres systèmes de pensée passibles de transmissions orales, voire écrites, fussentelles réservées aux heureux élus. Je vais m'expliquer. Sur le départ de son " "terrain" de référence " (les Grassfields du Cameroun de l'ouest), rapporte notre ethnologue, certain geste d'un haut personnage le médusa.

«En 1974, au moment de quitter le Cameroun après deux ans de présence, je rendis visite au chef [d'un] petit village [...] afin de prendre congé [...]. L'entretien terminé, il me raccompagna jusqu'à la limite de son hameau. Arrivé là, il s'arrêta [...]. J'étais debout devant lui. Il prononça quelques mots d'adieu, me donna une chèvre et, sans crier gare, cracha sur moi à plusieurs reprises. J'étais saisi de stupeur mais n'en montrai rien. La prudence m'ordonnait de ne pas faire disparaître la précieuse salive dont ma chemise était maculée. Je pris congé. » (CC:59).

Il lui fallut attendre quinze ans pour qu'un tel comportement, de prime abord assez étrange, prît sens au regard d'un ensemble de faits, rendu consistant par sa réflexion sur la gestuelle, les techniques du corps, les conduites motrices, agencées enfin comme " pratiques, techniques de soi, actions de soi sur soi et sur les actions des autres » (CC: 59). Qu'est-ce à dire ? Retournons sur les lieux. Makon, une chefferie des Grassfields, fin décembre 1984 : les greniers sont pleins. Le chef va festoyer, «nourrir son peuple » en protagoniste d'une "propédeutique dansée " scrupuleusement décrite dans CC (pp. 61-63 et suiv., passim). Lors de la cérémonie, une fois sa corne à boire emplie de vin de raphia par un serviteur, le corpulent dignitaire la porte à sa bouche, puis crache ce précieux breuvage ainsi mêlé de salive en le pulvérisant sur les autres danseurs et danseuses courbés pour s'imprégner de la substance éminente.

La culture matérielle et la mise en objets? Nous y sommes. Le chef est un récipient par excellence. À telle enseigne que Warnier surplombe maintenant ses observations 
initiales en y voyant, pour faire bref, une "dynamique des vases communiquants" (CM : 63-64; il a le sens de la formule). C'est que, dans la population intéressée, chaque personne ou catégorie lui parait subjectivée en termes, mieux, en matières et formes, de récipients précisément; des récipients reliés. Partout ces contenants, avec leurs entrées, leurs sorties : bouche humaine, ouvertures d'artefacts (un seul vocable désigne l'une et les autres) qui incluent celles, fort exiguës, des cases, d'où la gestuelle délicate imposée pour y passer; puis les groupes d'habitations au cœur desquels se trouve un palais, et constituant le type même de ce système de "vases " que font communiquer, en pareil cas, des loges, conduits, cours, chemins, valves, sas, etc., et où le sujet se déplace en suivant des itinéraires définis par son sexe, sa condition sociale... Nombre d'exemples circonstanciés sont fournis (blâmes quant à la déperdition du "contenu » des notables, pratiques successorales, sexualité, etc.), qui montrent comment l'auteur entoure à présent, d'un large mouvement, la totalité de données jusque-là décousues. Données précédemment disparates... ou muettes, l'interprétation ne pouvant rendre raison des énoncés obscurs ou lacunaires entendus sur place, lesquels ont dû céder la parole, si j'ose dire, aux corps-sujets et aux conduites qui, eux, sont saturés de sens, pourvu qu'on sache le débusquer. À en croire Warnier, sous ce jour nouveau, c'est aux outils d'analyse de Parlebas qu'il doit faire droit. Tous les "stéréotypes moteurs" invoqués au fil du chapitre "sont intégrés par des personnes différentes», mais «pris un à un ", comme résultats des aptitudes qu'exerce chaque individu pour élaborer, seul, ses conduites spécifiques, ils relèvent de la psychomotricité. En interaction motrice, plusieurs agents sont engagés dans la sociomotricité, qui «implique la communication motrice ou sémiomotricité » (CC: 62-63). Dans ces conjonctures variées, grâce à la régie inédite du spectacle représenté, toute individualité que subjective la culture matérielle des contenants et contenus connectés exhibe, à quelque degré, sa nature construite de récipient animé, recevant ou déversant diverses essences. Au premier chef, le chef qui, pendant la chorégraphie cérémonielle évoquée plus haut, active semblables significations en dispersant vers ses gens son propre contenu (de là, l'heureuse expression de "propédeutique dansée ») : celui-ci, passé par l'alambic qu'est l'insigne personne, ayant ainsi acquis un prix sans pareil, circule parmi le peuple comblé. Au même instant le détenteur de tels pouvoirs les reproduit-il en agissant sur lui-même et sur l'action des autres par re-mise en objets interposée.

Que je surinterprète ici des interprétations, ce n'est pas à moi qu'il revient d'en juger, mais aurais-je interprété une surinterprétation? Assurément, lorsqu'un objet est caché à tous les yeux, il faut bien l'inventer pour pouvoir le découvrir, comme l'écrit Sartre (ou à peu près; manière de tirer du réel hors de l'ombre). Toujours est-il que la démarche de Warnier, d'une hardiesse séduisante, expose à quelques risques. Mais faisons le départ entre ceux-là et un problème général.

«Personne ne "prouvera" jamais que $\mathrm{W}$ a eu tort en affirmant que l'histoire de l'humanité est l'histoire de l'échange, ou que $\mathrm{Z}$ a eu raison de comparer un culte de possession à la Commedia dell'Arte. » (Olivier de Sardan 1996 : 38).

De même, qui voudrait administrer les "preuves" de la validité des "hypothèses Grassfields " sera à l'évidence bien en peine d'en trouver certains moyens expérimentaux équivalant aux protocoles des disciplines « dures ». Cela étant, aucune pratique scientifique, pas une, ne fournit de résultats à tout jamais irrévocables (les maths restant peut-être hors concours). Et les productions des sciences douces semblent bien plus problématiques : beaucoup sont immunisées contre les démentis empiriques. De sorte que pour la présente discussion, mieux vaudrait parler de 
conjectures ou d'une heuristique. Au surplus, tous les anthropologues ont vu ce qu'ils décrivent, mais décrivent ce qu'ils veulent voir, c'est inévitable malgré l'«attention flottante " parfois ambitionnée dans l'enquête. Voir, ou entendre: là pourtant, si l'ethnologue n'a pratiquement jamais entendu de discours sur l'idéologie incarnée dans les sujets, les formes et les actes que l'on sait, son bon vouloir n'est pas en cause, puisque «En vingt ans de présence intermittente sur le terrain, [il n'a] jamais rencontré personne qui dise explicitement : "le notable est un récipient" ", affirme-t-il avant de méditer tous ces non-dits (CC:65) ${ }^{19}$. Néanmoins, quand il put voir ainsi les choses et les êtres, ce fut, selon lui, parce que « ...un texte ronéoté, écrit par deux prêtres catholiques bamilékés soucieux de montrer que le "culte des ancêtres" n'était pas incompatible avec la foi catholique », lui avait permis de "trouver les mots pour le dire » : dans ce document, le notable est regardé, au pied de la lettre, comme « "une espèce de tirelire vitale de toute la famille" [etc.]» (cité in CC: 65, ital. de Warnier). Édition princeps ou prolégomènes d'une théorie de la production grands hommesrécipients? Je n'insinue rien de vénéneux ni ne veux jouer les donneurs de leçons dans cette question et la suivante, nous savons tous que d'une étincelle peut jaillir la lumière. Mais face à des « informateurs privilégiés » ou des exégèses déjà proposées dont celles d'autres ethnographes-, l'anthropologue devant, plus souvent qu'à son tour, faire son ordinaire de l'interprétation d'interprétations, la critique des sources n'est-elle pas, en ce cas-là, plus que jamais nécessaire?

31 N'empêche, rechercher du sens dans les idéopraxies plutôt que dans les idéologies ouvre bien la voie à l'intelligence de la société des Grassfields. D'autant que les malentendus procèdent quelquefois du mensonge institutionnalisé, essentiel à l'entente au sein de certaines communautés et au maintien d'un étranger dans la bonne distance ( $c f$. La Cecla 2002). Les idées, les mots et les choses n'ont ni même statut ni même rendement pour l'analyse, c'est notoire. À un grand sculpteur qui lui disait «J'ai quelques idées de poèmes ", un poète tout aussi grand rétorqua : "Maître, on ne fait la poésie avec des idées mais avec des mots ». Et une société n'est pas faite qu'avec des mots et discours, elle l'est surtout avec les actes des sujets, s'exclamerait Warnier (j'imagine).

"Tous [ceux qu'il observe] ont les mêmes symboles en tête. Aucun n'a les mêmes gestes dans le corps.» (CC: 62). "Ces actions [de soi sur soi et sur les autres] permettent de faire et de défaire les sujets, voire de les détruire, en communiquant et en "pensant avec ses doigts" [...], sans qu'il soit nécessaire d'en parler. [...] le discours unanimiste est brutalement démenti par les pratiques inégalitaires, et repose justement sur un non-dit. Il permet le tour de passe-passe par lequel ceux qui ne contiennent rien, et en sont bien persuadés [...], admirent, aiment [...] les notables dont ils reçoivent tout [...] parce que ceux-ci les ont privés de substance, et entreposent dans leurs corps et leurs récipients incorporés tout ce qui peut l'être. Ainsi, les pratiques hiérarchiques et inégalitaires revêtent l'évidence et la nécessité des choses corporelles, matérielles, voire utilitaires. » (CC:81, mes italiques).

Que je sache, cela s'appelle aussi "dénégation", "distinction", incor-poration du "capital symbolique». Mais il y a ce surplus heuristique selon lequel chaque être humain singulier se dévoilera dans sa subjectivation par les artefacts, culturellement variable. Si bien qu'après semblable épiphanie, on devine l'honneur ambigu que fit à l'ethnologue son noble vis-à-vis en lui crachant dessus.

Médiations diversifiées

Si la mise en objets telle que l'expose Warnier pour les Grassfields -il la met en intrigue - apparaît éclatante par quelques côtés, la longue seconde partie de son livre réfracte 
un discours éclaté; et prolixe ${ }^{20}$. Non que sa lecture soit fastidieuse, loin s'en faut, mais son agencement même, lequel en rend la synthèse malaisée, provient sans doute d'atermoiements dus à un brusque changement d'échelle. Ce qui m'impose de prendre du champ, par contraste, et de rendre compte sans vétiller.

La première partie a proposé «...une théorie [applicable] quelle que soit la soit la société considérée ", écrit l'auteur (CC:87). Et de poursuivre :

«Par contre, il s'agit [maintenant] de se pencher sur un seul type de société, le nôtre. Au regard de la production du sujet par la mise en objets, quelles sont les spécificités de nos sociétés industrielles et marchandes?» (loc. cit., ital. dans le texte).

Or, les propriétés de "nos sociétés " semblent le contraindre à les passer aux cribles pour le moins dissemblables de leur "institution imaginaire" (Castoriadis), de la psychanalyse (le « désir »), de l'«approvisionnement de masse » et de la "théorie du déchet ", des conceptions marxienne et bourdieusienne, sans compter Merleau-Ponty bien sûr (avec son "sujet incarné »), Hegel et quelques autres. Flèches sont faites de tout bois.

36 C'est qu'aujourd'hui, « la mise en objets [...] a un point de passage obligé : l'argent et le marché [...]. Si donc la subjectivation est médiatisée par la culture matérielle, celle-ci [...] est médiatisée par l'argent » (CC:88). D'où vient que de nouveaux problèmes se posent, et que soient arpentées des voies multiples pour tenter de les résoudre. Les «styles de vie » étant des «techniques de soi » (cf. Foucault), en économie marchande, la consommation constituera un élément de la "production de soi par soi ». Mais dans ces structures où est prise la dépense monétaire, s'interpose, entre l'homme et l'objet, l'action du développement technique, des médias et des "industries de l'imaginaire ». Va-t-il en découler l'aliénation par la valeur-signe et la valeur d'échange des marchandises, obstacles à l'assomption de la liberté ? «L'imaginaire du matériel » (chap. 4) prend « imaginaire» dans des acceptions plutôt distinctes, qui convoquent tour à tour Bergson, Sartre, Castoriadis, Lacan, Pierre Kauffmann, Gilbert Durand... Épiloguer sur cette fresque polychrome excéderait mes capacités. Par bonheur, Warnier fait le point.

«Bachelard [et la plupart des susnommés] revalorisent l'imaginaire comme capacité à produire des images matérielles, sans pour autant expliquer d'où vient cette capacité et quels en sont les mécanismes. C'est du côté de la psychanalyse qu'il faut se tourner pour trouver les pulsions, le fantasme, et son articulation au réel et au symbolisme [mes italiques : clin d'œil à Lacan, je suppose - J.-L. J.], les mécanismes de la production imaginaire. Castoriadis montre à juste titre que cette production imaginaire, individuelle dans ses origines, fait l'objet d'une institution sociale.» (cC:107).

S'ensuit l'exemple ethnographique de la Sape "comme technique de soi », lequel illustre l'institution sociale de l'imaginaire matériel à l'époque moderne (CC : 108-112)... SAPE : «Société des Ambianceurs et Personnes Élégantes ", tel est le label d'un groupe informel rassemblant des jeunes gens déscolarisés originaires du Congo-Brazzaville, qui, au tournant des années quatre-vingt, s'aventuraient à Paris où la MEC (« Maison des Étudiants Congolais ») les prenait en charge. Ces "Sapeurs », dandys d'exception étudiés par Justin-Daniel Gandoulou (1989), se lançaient à corps perdu dans une entreprise de subjectivation complète et délibérée. Aux dépens d'acquisitions que nous jugerions de première nécessité, ils amassaient «la gamme»: certains articles considérés de rigueur, tenues bon chic «hauts de gamme» justement, aux griffes 
prestigieuses (des inspections mutuelles et formalisées, en ce bel appareil, avaient lieu à la Bastille)... Toilettes dont ils s'endimanchaient afin de parader, dès leur retour d'émigration, voulant de la sorte persuader leurs congénères restés sur place d'un changement radical. Car il y a plus: le "teint parisien" comme la corpulence appropriée s'obtenaient en usant de cosmétiques et d'aliments féculents. Ainsi ces sujets se construisaient-ils " par la consommation de l'altérité » $(C C: 111)^{21}$.

Relevons au passage que jouent ici, c'est évident, des représentations prééminentes qui tracent, du point de vue des dominés, un modèle corporel exogène et surenchéri; mais aussi, qu'en réciproque biaisée, les figures des (ex)colonisés sont perçues par leurs spoliateurs des mondes dominants (ou l'ont été, cf. Boëtsch \& Chevé 2000) sous le prisme d'un échange inégal d'altérités et de stéréotypes ambivalents. Contreparties boiteuses qui devront spécifier l'un de nos grands thèmes de recherche, dès lors que l'anthropologie de l'exotique «...no puede escapar de ser el estudio de las relaciones entre la cultura occidental y las demás » : ne peut s'évader de l'étude des rapports entre la culture occidentale et les autres (Terradas $1988: 158$ ).

Dans le cas des Sapeurs, lesdits rapports exercent leurs effets et se renforcent par le truchement de la consommation, «...technique de production de soi [fusionnant] le sujet et son apparence [tout cela au travers] d'objets matériels qui font image, [...] dans un imaginaire qui, en tant que fantasme, participe d'une certaine illusion...» (cC:111). Or les analyses de la consommation comme fait social accusent un retard extrême, dixit Warnier (CC: 113). Partant, son chapitre 5 («La mise en objets par l'approvisionnement de masse ») présente un historique circonstancié du problème et s'achève sur le fin du fin. Derechef, je fuis lâchement la revue de détail des théories antécédentes (Thomas d'Acquin, Veblen, Elias, Bourdieu, Baudrillard, Mary Douglas...) pour évoquer ce nec plus ultra : l'idée de sys-tème d'approvisionnement, que l'on doit au livre de Ben Fine \& Ellen Leopold (eds, 1993). Consommation de masse opposée à la production, attitude du consommateur (ou du producteur), voilà bien des notions fallacieuses. Elles ne sauraient désigner de réalités identifiables, cohérentes, se trouvent désagrégées: autant d'objets partiels que de sciences qui les étudient, de méthodes qui les isolent. Mais si l'on coupe plus fin à l'effet de rebâtir un seul édifice, alors il surgira, fait de multiples pratiques, "globalement structurées par des systèmes d'approvisionnement dont les profils varient considérablement de branche à branche " (CC:129, mes italiques). C'est que sous l'optique des deux auteurs cités, chaque « facteur horizontal» (suivant leurs propres termes) figure soit le registre d'une discipline particulière, soit les variables privilégiées d'une approche de la consommation, et traverse des verticales: les classes de biens ou services, réparties en filières. Là où se croisent lignes et colonnes, des traits distinctifs pondèrent les facteurs horizontaux dont tel ou tel sousensemble, ainsi étalonné en degrés de pertinence, constitue un système d'approvisionnement pour une branche d'activité définie (une filière; $c f . c C: 127$ et suiv., tabl., ex.). N'en disons pas plus; car, en clair et au bénéfice du présent propos, tout cela signifie que l'on ne peut retrancher nulle marchandise de son entier contexte choses, réseaux, agents, groupes-, mais qu'aucun type de consommation -automobile, meuble, vêtement, éducation, etc.- ne doit s'analyser comme d'autres. Au reste, Warnier ne conclut guère davantage, sinon en réintroduisant la dynamique incorporée par les sujets.

« Il en résulte que les régimes de subjectivations matérielles... » [réfractées " par de nombreux paramètres de classes et de conditions »] «...sont extraordinairement divers. Les techniques de soi et les moyens d'action sur des actions par 
l'incorporation de la culture matérielle dans les conduites motrices n'ont sans doute jamais été aussi diversifiés qu'ils le sont aujourd'hui.» $(C C: 129)^{22}$.

Tout plaide donc en faveur des enquêtes ethnographiques sur la relation sujets-objets matériels au sein de nos sociétés contemporaines. Les travaux de Daniel Miller (1987) ou, spécialement, d'Arjun Appadurai (2001), en fournissent de brillants témoignages; celui-ci jette des passerelles neuves entre local et global, cultures du corps comme du sport comprises (cf.supra, n. 7), dans les paysages redessinés d'une «mondialisation » dont il met en cause les topiques. Pendant que pour Warnier, laudatif envers Appadurai et Miller, le grand dispensateur d'idées, c'est Michel Foucault : seul capable de prendre en compte «l'action bien réelle du sujet sur soi et sur les autres, la manière dont il se gouverne, ce qu'il fait de lui-même, et comment cela affecte les autres sujets » (CC : 134), le philosophe du Souci de soi nous lègue en ces termes-là, ou à peu près, sa définition de la " gouvernementalité ", concept capital pour notre auteur. De sorte que l'« approvisionnement » se révèle, à ses yeux, mode spécifique de gouvernementalité (cf. CC : 135). Ce qui ne l'empêche pas de revenir à Marx -et à l'argent.

41 Et nous voilà ramenés à la «Médiation par l'argent comme modernité " (sixième et dernier chapitre). Après l'entremise de quelques classiques (valeur d'usage/valeur d'échange, Simmel, Kopytoff, Polanyi...) et le rappel de certaines recherches sur lesquelles il serait oiseux de s'arrêter car les synthèses ou commentaires concernant l'autre ouvrage, $A M$, valent pour leur fond, on en arrive d'abord à l'embodiment. Cette notion, employée par des ethnologues anglophones, s'inscrit dans la problématique des représentations, des symboliques, des pratiques aussi, touchant à la «production du corps " en rapport avec les distinctions de genders, leurs substances, etc. (Les noms et livres de Marilyn Strathern, Annette Weiner, Maureen MacKenzie sont mentionnés; mais qui, en France ou ailleurs, pourrait oublier Françoise Héritier, Bernard Juillerat, Sylvie Fainzang, David Le Breton, Colette Guillaumin...? Qui omettrait des ouvrages qu'ont dirigés Maurice Godelier et Michel Panoff... ?) Néanmoins, Warnier remet les points sur quelques « $\mathrm{i}$ ». Pour sa part, il entend bien « ...articuler l'embodiment avec les conduites motrices des sujets, dans un théorie générale du rapport à la culture matérielle" ( $C C:$ 147). Puis, une fois posé le «paradoxe de la marchandise authentique $»^{23}$ (cf. notamment Julien 1994 in Warnier, ed., 1994, sur la vogue exotique des meubles chinois fabriqués à Paris pour le marché français et pour ses goûts, fort différents de ceux des Célestes), ce chapitre ultime aborde la théorie thomsonienne du déchet (Thomson 1979). Devinette : « "qu'est-ce que le pauvre expulse et jette à terre, et que le riche emballe précieusement pour le mettre dans poche? Réponse : la morve" " (CC: 156). On se distingue par la manière de traiter le rebut. Les classes sociales produisent différemment la valeur des choses. Un même artefact (voire «naturefact ", dirais-je) relèvera des catégories «biens éphémères » ou «biens durables » suivant ce que décide "la société »; son statut «se calque sur celui de la classe qui s'y met en objets » (cc : 157).

Et in fine, l'abondante seconde partie signale que ni l'argent ni le marché n'introduisent de « distanciation spécifique entre le sujet et l'objet au regard de l'action motrice ». Où réside alors le changement face aux «sociétés de la tradition »? En ceci : l'argent, le marché « et l'inégale répartition de la richesse et du pouvoir diversifient les [modes de] subjectivation par la culture matérielle en fonction des classes, des catégories sociales et des revenus » (CC:160, mes italiques).

« Dans la mesure où les membres de ces différentes catégories pensent avec leurs corps, leurs doigts, et les objets, ils pensent différemment, et, bien souvent, ne se

Techniques \& Culture, 39 | 2002 
comprennent pas. Les modalités de leurs subjectivations respectives sont trop éloignées les unes des autres. Ces sujets ne sont pas du même monde, ils ne sont pas faits du même bois. » De plus, «À revenu égal, les systèmes d'approvisionnement, les techniques du corps, [lesdites] modalités ne sont pas les mêmes aux États-Unis, au Japon et en Allemagne. » (loc. cit.). cousues, parfois, en manteau d'Arlequin. Certes, invité dans le sérail qu'elles habitent, j'ai beaucoup appris (comme à des cours magistraux) : l'auteur en connaît les détours. Mais pourquoi pareil éclectisme? Parce que le chercheur ne sais plus où il va? Qu'il se noie dans l'océan de son érudition? Pas du tout, les raisons en tiennent d'abord, fatalement, à ces resserrements du champ que j'ai sous-entendus tout à l'heure : l'échelle a changé. Par nécessité, l'anthropologue embrassait l'«hominisation » d'un regard vraiment très éloigné, qui permettait d'apercevoir en grand angle les données biologiques, primatologiques, culturelles, technologiques, socio-écologiques et leurs connexions hypothétiques, de surcroît dans la durée ${ }^{24}$. L'interprétation relative au terrain ethnographique "exotique " s'est satisfaite de la distance moyenne, le plus souvent de règle en l'espèce. Mais au présent de l'« endotique", le contraste est saisissant. Plus guère de raccourcis possibles, tout est de facto trop proche et parait tellement compliqué -tant il y a de médiations à répertorier (était-il annoncé). Quoique la fin du parcours redevienne assez simple, et c'est bien ce qui surprend. Les techniques du corps, la culture matérielle et ses mises en objets varient avec les situations de classe, en même tant que selon les pays comparés... Peut-être le rasoir d'Occam aurait-il trouvé à s'employer ici.

Je veux pas jouer les professeurs, mais seulement dire que l'on doit maintenant réfléchir aux étiologies restantes d'une difficulté campée devant nous tous, et se réfléchir soi-même. Techniques, culture matérielle, efficacité, et "sujet", sont-ils ou non des concepts problématiques dont l'extension est, elle aussi, variable, comme leur compréhension? Les "interprétations ", façon herméneutique et métaphores, sontelles de notre ressort -ou la prospection des causes efficientes ? Les unes et les autres? Ceci encore : d'aucuns, on y revient, seraient la proie d'une " préoccupation lancinante pour l'action efficace sur la matière ", ai-je écrit en citant Warnier tout au début. Selon lui, le rapport privilégié de l'homme à la matière affecte tous les aspects de l'êtrehomme (c'est incontestable), l'outillage n'étant «qu'un cas parmi d'autres qu'une civilisation moins entichée de technique que la nôtre mettrait à sa juste place : pas la première » (c'est à voir).

En premier lieu, il faut circonscrire, même très schématiquement (tenez, par un schéma), les catégories respectives qui tendent, selon moi, à spécifier les démarches propres, disons, à deux groupes de chercheurs, et non des moindres: "Matière à Penser ", « Techniques et culture ». 

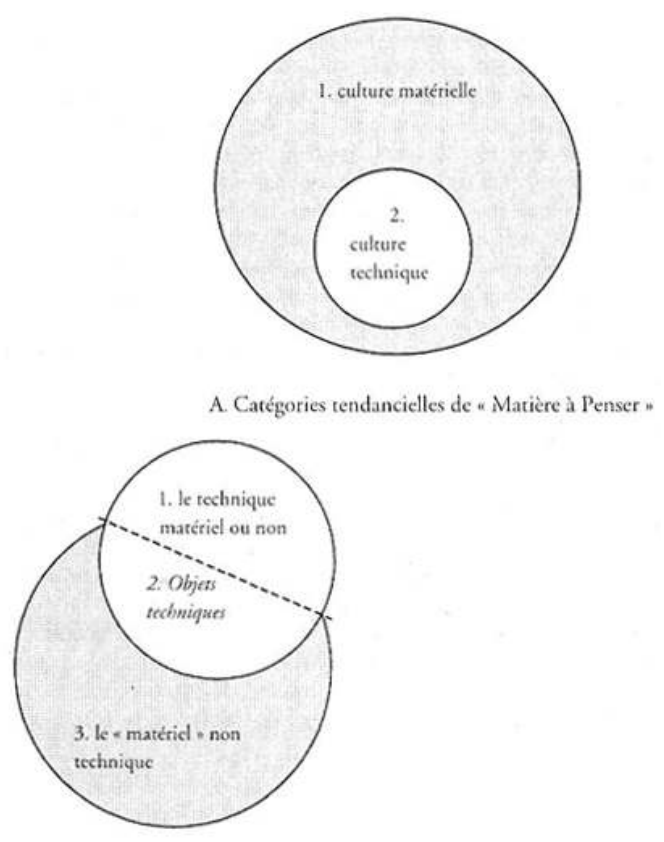

B. Catćgories tendancielles de - Techniques et cuiture *

47 A-1, A-2 : la culture matérielle (en général) déborde largement la culture technique (matérielle); cf. tout ce qui précède (et lire au plus vite $C C$, puis $A M$ ).

B-1: le technique, matériel ou non. Composition: les dispositions cognitives invariantes, les «théories locales » variables, les savoirs, les savoir-faire, les instruments qui ont pour effet de rendre la matière, la nature « consommables » (elles sont du même coup pensables, en tout cas pour Homo)... ou de détruire celles-ci.

B-2: les objets techniques (sous-ensemble de B-1). Matériels, ils consomment, transforment ou détruisent, médiatement ou non, la matière, la nature. Mais sont-ils « faits pour »? Surtout, ils « servent en... » : un couteau sert en coupant (cf. Sigaut 1991). Concrètement, aucun objet n'a de propriétés que purement techniques. «Un objet seulement technique n'est qu'une utopie » (Latour 1992: 8) ? Peut-être pour la phénoménologie. Mais pour quelques méthodes, pour certaines ontologies, c'est a minima une abstraction. Abstraction fonctionnelle ou fiction utile. Une « dimension » possible de (presque) tout objet (parmi ses autres aspects : esthétiques, etc.). Un « service » de l'objet tangible (le concret, c'est de l'abstrait rendu familier par l'usage, disait le physicien Paul Langevin). Toute « machine » est sous ces rapports-là un objet technique. Les appareils constitutifs de l'organisme, non. (Fonctions premières détournées : ready-made à la Marcel Duchamp.)

B-3 : le «matériel » non technique. C'est tout le reste de la «culture matérielle » (cf. A : A-1 moins A-2); en ce sens, il peut s'agir des produits (intermédiaires ou finals) de la matière "consommée ", à destinations esthétiques, rituelles, etc. Il arrive que du «matériel non technique » «serve en faisant... » (cf. ci-dessus) : je me sers parfois d'une robuste statuette de la Vénus de Milo pour enfoncer des clous (je pense aussi avec mes doigts, mais en ce cas-là, mal, et ça fait mal).

51 Questions: Où se situe le corps? Sous l'angle "techno-logique », il est engagé dans l'action technique, et à la fois, matière d'œuvre modelée par cette action; à ces titres, suivant les choix de méthode, on le domicilie soit dans B-1 -agent-patient des « techniques d'objet »-, soit dans B-3 - 
agent-patient des «techniques de soi » (merci au "MàP»). Et le sujet ? Les techniques et plus généralement tous dispositifs et artefacts (B) étant dans la culture, elles font partie de son mobilier ontologique, constitutif de tout être humain. Dès lors, un sujet sera là aussi construit par les options d'une recherche: "sujet agent-patient des techniques de soi », acteur social, individu, organisme, en fonction des points de vue.

Mon épure parcimonieuse, qui n'engage que son concepteur, est révisable à tout moment, outre que je suis sérieusement demandeur de critiques constructives (inutile de me dire qu'elle laisse en sous-main le symbolique, les structures et faits sociaux, les rapports de production, vagues filigranes occupant le grand périmètre quadrangulaire, pour l'instant). Au dedans, on trouve "techniques et culture", mais pas de summa divisio. Les unes sont dans l'autre, et dans le social. Partant, en aucun projet général, l'étude des premières ne peut faire l'impasse sur leurs implications au sein du reste de la seconde; dommage que la réciproque ne soit pas flagrante pour tout le monde (les milieux «naturels» entrent en ligne de compte presque par définition car jamais phusis, la Nature, n'est donné absolu ou Nécessité pure, anankè). Ces liens intraculturels ne se valideront qu'au prix de confrontations intercul-turelles traversées d'une dimension historique. Nombre de travaux l'attestent, rien moins que « technicistes » et d'autant mieux comparables qu'ils sont adja-cents car venus d'un même corps de chercheurs. Quand Martine Garrigues-Cresswell et Béatrice Lecestre-Rollier (2001) montrent l'interdépendance de la gestion des risques, des complémentarités socioéconomique, technique et écologique «verticales " dans le Haut Atlas marcocain; lorsque François-René Picon (1999) établit l'assise d'une ethnohistoire comparative des effets différentiels de l'introduction du cheval aux Amériques -emprunt technique révélateur s'il en est-, ils concourent fructueusement aux avancées de ce dessein (outre Christian Coiffier, Sophie Desrosiers, Anie Montigny)... Tout comme le fait Geneviève Bédoucha (1987) qui, pour sa part, examine les tenants et aboutissants du contrôle social autant que technique d'une ressource décisive, l'eau, dans une oasis du Sud tunisien (et ces temps-ci, son appropria-tion, au sujet des étangs brennais du Berry). Dans une veine différente mais dans le creuset commun, Aliette Geistdoerfer reconduit mon propos vers l'« incorporation »:

«Le futur marin doit d'abord amariner son corps, afin d'acquérir l'équilibre à bord d'un bateau qui est tout le temps en mouvement [...]; formation [...] si importante que de retour à terre le marin doit réadapter tout son corps [...]. » (Geistdoerfer 1999 : 157; de même eau bien qu'au fil d'autres courants, $c f$. les écrits de Jacques Ivanoff -e.g. 1991).

53 Les techniques du corps doivent, elles aussi, s'analyser en tant que techniques d'objets incarnés, en relation avec "tout le reste ", et cela requiert d'identiques exigences comparatistes, fût-ce par des rapprochements commencés à titre d'aile. Quelquefois, une culture entière semble régir les choix d'objets qui sont conçus en fonction de postures qu'ont intériorisées les hommes - ou les femmes (cf. l'exemple de l'«iler » en Afrique, qu'étudia Henri Raulin; curieusement, dans AM, avec tant de contributrices, point de distinctions par genres). Mais ce n'est pas tout. Ainsi Junzo Kawada (1996) compare-t-il les façons de marcher, de s'asseoir, de porter, etc., propres aux Japonais, aux Africains de l'Ouest, et aux Français dans certaines traditions, en y reliant d'autres gestes ethniquement motivés (ce que, parlant du vêtement, Haudricourt avait esquissé : 1987a). Lévi-Strauss évoque quelque part (mais où? je le paraphrase de mémoire) la civilisation japonaise en ce qu'elle figure, avec la nôtre, des rapports de symétrie inversée : le Japon et la France sont situés aux deux bordures, est et ouest, de l'immense 
espace eurasiatique. D'où l'idée, qui ne saurait étonner venant du maître structuraliste, que l'on repère à l'un et l'autre de ces pôles les degrés permutés d'un ensemble de transfor-mations, comme en miroir. Or, en fait de techniques du corps, de culture matérielle (notamment), il a un lointain et surprenant devancier, Luís Fróis (1993). Ce Jésuite acheva en 1585 un Traité répertoriant plusieurs centaines de couples d'opposés qui soulignent les antinomies des mœurs nippones avec celles, surtout, du Portugal, pays natal de l'enquêteur comparatiste ${ }^{25}$. Nous devons aller plus avant dans les voies frayées de la sorte, en tous lieux, en tout temps, sur tous les registres, jusque sur la gamme des "techniques sexuelles», comme Mauss l'avait suggéré (pour l'ExtrêmeOrient, $c f$. déjà Van Gulik 1997 et Shimekawa 1997). Au four et au moulin, donc, à voir si l'habit fait vraiment le moine ou celui-ci fait celui-là : que ces leitmotive nous pilotent. Et j'y pense : habeas corpus? -que non! On n'a pas un corps, on est son corps, écrit quelque part Jean-Pierre Warnier (dans CC). Bon, mais c'est selon les états de conscience. Le dualisme du «j'ai un corps et puis j'ai un esprit » n'a rien d'une vieillerie ethnocentrique caractérisant le sens commun des Modernes ${ }^{26}$; dans plus d'une culture, il est certains moments du vécu de l'apprentissage ou de telle grave épreuve -le combat préparé où le guerrier doit s'avérer sans ambages efficace- qui « ...feront passer l'homme d'être un corps à avoir un corps ", comme l'écrit justement Nathan Schlanger (1991: 125) à propos des réflexions de Mauss sur les rapports entre sociologie et psychologie (1985a).

En second lieu maintenant, cette "efficacité-critère distinctif ", douloureuse obsession paraît-il, Warnier s'en fait le contempteur. Avouons-le, ce n'est pas le seul chercheur à qui elle pose un problème ${ }^{27}$. Mais je ne vois pas non plus qu'il le résolve vraiment. Une manière de l'aborder par le travers, a contrario, consisterait dans l'étude de l'erreur et de l'échec techniques (entre autres domaines), considérés comme analyseurs aussi bien de processus cognitifs non conscients, transculturels, que des savoirs ou des systèmes épistémiques explicites, divers ou changeants, qui s'y rattachent par attentes intuitives et implicites interposées (sur ces notions, cf. e.g. Boyer 1997) : je me remets au rouet làdessus. Peut-être comprendra-t-on en quoi l'erreur, l'échec engendrent une rétrospection, prometteuse de rétroaction et donc d'innovation, sur des «actes traditionnels " ainsi révélés inefficaces relativement aux fins visées. Car énoncer cela apparaît nécessaire mais insuffisant: le "comment » reste à mettre en relief et en perspective. En revanche, à la différence de trop d'ethnologues, tablons déjà sur ceci : toutes les cultures, même très " exotiques ", admettent de fait dans leurs conduites les plus banales, leurs comportements quotidiens, quelques postulats d'efficience pratique envers la matière. $\mathrm{Y}$ compris quand elles ne tracent pas dans le discours nos démarcations à nous : «faits techniques, et autres »; « le travail, et le reste »... Il y a là des universaux de l'espèce -et le réel out there, qui travaille par l'intercession de nos outils (lato sensu) intellectuels et matériels. Mais aussi, conjointement à nos propres classements, on doit ranger ces variétés de leurs taxinomies à cet égard parmi les données d'un problème passionnant.

Troisièmement, donc, pourquoi accorder tant d'importance aux techniques ? Parce que «notre civilisation » en est éprise (cf. supra) ? Non, ou pas seulement. Il n’y a là rien de plus exorbitant ni d'inopérant que l'attribution de la première place au «sujet» rapporté par une vague "épistémologique » qui déferla au début des années 1980 avec les flux et reflux de l'idéologie (pour un panorama: Dosse 1997; animaux devenus "sujets »: $c f$. la philosophe Élisabeth de Fontenay, et Lestel 2001; $c f$. aussi le récent 
Kaufmann 2001). Dans d'autres civilisations, ailleurs ou jadis, ce concept, « sujet ", est (fut) difficilement pensable, ou pas du tout, Warnier le sait sûrement; de hautes cultures arabo-musulmanes eurent du mal à s'en accommoder. Et l'important est qu'en Occident, le sujet, au demeurant toujours diapré de reflets instables, n'existe pas de toute éternité, procède d'une origine, connaît une histoire. Il n'est que de songer, puisque Warnier s'y réfère, à l'herméneutique du sujet selon le "second " Foucault (cf.e.g. 2001). Si l'on me permet ce grossier synopsis, il s'agit, je crois, d'une sorte de généalogie de l'intériorité occidentale, ouvrant vers une expérience de soi anti-cartésienne (Descartes : autre penseur, autre pensée du sujet). Elle fonde une éthique ainsi qu'une politique par quoi l'homme se libère du "Pouvoir" et de sa "technologie", se gouverne lui-même : «techniques de soi », donc, et « gouvernementalités », techniques de pouvoir, conscientes... Qu'en est-il alors des techniques de soi par incarnation infraconsciente de la dynamique des objets? Sur ce chapitre, pour qui veut vraiment saisir comment elle s'incorpore, encore faut-il des recensions, des descriptions et des analyses approfondies de ces objets, de leur dynamique (e.g. Akrich 1995, Gelbert 1997, Willems 1995), et non de simples présupposés qui rebondissent sur le problème, puis, en fin de parcours, se retrouvent in statu quo ante: le verdict de l'empirie est capital. Certes pas seulement dans le domaine des techniques stricto sensu; mais ces dernières constituant toujours en bonne partie une boîte noire, il y a du pain sur la planche... D'autant que celles qui relèvent de l'anthropologie ou de l'histoire comparée demeurent mal connues, et que chaque jour en naissent de nouvelles, dont souvent la complexité s'accroît.

«Cela fait plus de deux siècles [...] que la technologie [i.e. l'étude des techniques] ne se développe que par à-coups, au gré de modes qui reviennent tous les quarante ou cinquante ans, au rythme peut-être des cycles techno-économiques, mais aussi chaque fois qu'une génération nouvelle redécouvre pour sa part l'importance de la question et l'ignorance dans laquelle elle a été tenue à son sujet. » (Sigaut, pp. 1-15 in Cresswell \& Sigaut, eds, 1987 : 1; à propos d'autre cycles, sur les relations entre faits techniques et faits sociaux, $c f$. Cresswell, notamment 1994 et 1996).

Mais revenons à l'argument principal. Ou plutôt au contre-argument: de toute évidence, si l'absence de la notion de sujet dans les conceptions, les sémantiques de certaines cultures, à certaines époques, n'est pas dirimante pour l'analyse de celles-ci en termes de "sujet» mis à la première place, alors, à moins d'aller contre cette évidence, il en est de même quant aux techniques, sauf auto-contradiction ou indifférence devant le sophisme tacite et trop commode du "deux poids, deux mesures ». Le sujet, la technique : personne n'« imagine » ces choses-là, on leur accorde notre attention, ce qui est assez différent. En effet, comment faire partie du paysage et en avoir la vue? Par la rencontre de celui-ci et de panoramas lointains, conjonction d'où naîtront un beau jour, en surplomb de tous ceux-là, des catégories unifiantes, comptables et de l'un, et des autres. CEuvrons donc en ce sens, faisant fond, qui sur les techniques (savoirs objectivés), qui sur l'objet subjectivant ${ }^{28}$.

Enfin, nous voici confrontés au dernier problème. Approches phénoménologiques du "sujet ", herméneutiques... Rien d'étonnant que j'en appelle ici à Husserl (Recherches logiques), car on y reconnaîtra quelques thèmes: description des "vécus » saisis en essence, retour aux choses mêmes qui sont ces vécus-là, en lesquels réside « l'apparaître de l'objet », à savoir les phénomènes. Et d'après Merleau-Ponty (1997), les apparences sont toujours enveloppées «dans une certaine attitude corporelle». Cela, dans le vaste mouvement de l'œuvre de très grands penseurs. Mais Heidegger : «Bäh! 
Die Wissenschaft denkt nicht!», la science ne pense pas ! -du moins la « vraie » Science-, pontifiait-il. Doit-on en inférer que celles qui pensent ne sont pas de vraies sciences? Bien sûr que non! « Ça pense » dans toutes les disciplines, comme par exemple le fait très bien Warnier en dégageant une heuristique de ses interprétations relatives aux Grassfields. C'est indispensable, pour peu que l'on répudie à temps le démon de l'analogie qui fait le lit de tant d'artifices. Cependant, il me semble qu'aux yeux des membres du MàP, toute approche réductionniste (même si elle pense) ne serait plus de mise. Or la réduction, tant décriée chez les anthropologues, réputés «antiréductionnistes " par profession de foi, a remporté quelques menus succès dans divers champs du savoir scientifique. En user, c'est aussi pouvoir mieux discerner ce qui lui reste irré-ductible, au moins provisoirement, et la rigueur viendra au rendez-vous. À ce titre, refuser d'envisager pour notre profit d'ethnologues des cultures maté-rielles, les questionnements, comme certains résultats, de la psychologie et l'anthropologie dites cognitives, n'est que répondre à quelques dogmatismes ou des a priori, par des préjugés dogmatiques «distinguant " trop facilement ceux qui prétendent vilipender ainsi les oripeaux de la mode. Certaines convic-tions sont plus dangereuses que l'esbroufe. Assurément, la psychologie évolu-tionniste, state of the art outre-Atlantique, brûle les étapes en voulant rejoindre au plus vite les sciences positives, et attrape parfois des moucherons avec un appareillage digne de la guerre des étoiles (c'est le cas de Pascal Boyer, $c f .2001$ surtout). Mais pourquoi ignorer ce qu'elle ambitionne?

« Evolutionary psychology is simply psychology that is informed by the additionnal knownledge that evolutionary biology has to offer, in the expectation that understanding the process that designed the human mind will advance the discovery of its architecture. It unites modern evolutionary biology with the cognitive revolution in a way that has the potential to draw together all the disparate branches of psychology into a single organized system of knowledge [...] Culture is not causeless and disembodied. It is generated in rich and intricated ways by information-processing mechanisms [that] are, in turn, the elaborately sculpted product of the evolutionary process. " (Cosmides, Tooby \& Barkow 1992: 3, mes italiques). "We suggest that [the] lack of progress, [the] "failure to thrive", has been caused by the failure of the social sciences to explore or accept their logical connections to the rest of the body of science - that is, to causally locate their objetcts of study inside the larger network of scientific knowledge. [...] In place of [...] the Standard Social Science Model, there is emerging a new framework that we call the Integrated Causal Model.» (John Tooby and Leda Cosmides, pp. 20-136 in Cosmides, Tooby \& Barkow 1992 : 23-24, mes italiques; et l'« individu ", l'atomo, dans ce panorama ? Soit l'organisme avec son "esprit-cerveau », soit, pour un Dan Sperber [e.g. 1996], des entités infra-individuelles : idées ou représentations mentales « contagieuses »).

À vrai dire, de pareils objectifs me semblent confinés dans de lointains horizons; cela n'empêche nullement d'en découdre avec les explications causalistes - pas seulement pour les anthropologies qui briguent un statut « vraiment » scientifique-, ni de tendre à faire un sort au découplage causal entre nature et culture afin d'y substituer des principes-ponts. Une des voies ouvertes en ce sens est empruntée par la préhistoire, la paléontologie humaine, et certaines linguistiques ${ }^{29}$, nourries des apports de la primatologie moderne, comme l'indique Warnier au début de son livre (CC); par exemple, si l'on suit Dominique Lestel :

"Chez les animaux, même s'il [Leroi-Gourhan] ne l'évoque jamais en ces termes, les “animaux communicants" (oiseaux, mammifères marins) diffèrent en général des

"animaux techniciens" (chimpanzés, d'autres oiseaux, les insectes sociaux). L'humain est au croisement de ces deux lignées. C'est une spécificité remarquable, qui est cependant rarement revendiquée en tant que telle. De ce point de vue, 
l'humain n'est pas en dehors de l'animalité; c'est au contraire celui qui est le plus animal de tous. » (Lestel 2001 : 302-303). l'avait déjà pensé autrement.

«Il paraît [...] probable que le langage s'est appliqué préférentiellement à [des] secteurs d'activité [autres que la production des outils] (relations interindividuelles, notamment) et que l'apprentissage des techniques s'est réalisé principalement [...] sur le mode imitatif jusqu'à une époque récente (autour du paléolithique supérieur). L'hominisation serait donc aussi la longue histoire d'une jonction entre le langage et la technique... » (Guille-Escuret $1994: 292$, « Thèse 39 »).

$60 \mathrm{Au}$ surplus, un chercheur en neurophysiologie avance cette hypothèse encore hasardeuse, mais non sans quelques arguments et présomptions de preuves :

«...le perfectionnement de la commande des mouvements balistiques est peut-être à l'origine du langage, de la musique et de l'intelligence. [Ces actes] exigent une surprenante capacité d'anticipation [les corrections improvisées étant impossibles]. $\mathrm{Si}$ les mouvements phonatoires dépendent [de la même structuration neurocérébrale] », «Le don de la parole aurait été un avantage secondaire [side-effect] [de l'avantage balistique]. » (Calvin $1994: 114-115)$.

61 Voilà que la parole est dérivée du geste...

62 Tout cela invite à une entreprise qui conduirait un beau jour plusieurs sciences, humaines ou non, à se réunir autour d'un monisme de bon aloi, en contraste tranché avec certains pré-requis et découpages du réel glosés ci-avant; mais qui ne sont sûrement pas mis au goût de l'ontologie latourienne -Bruno Latour, d'esprit aussi agile que sa plume est véloce, grand pourfendeur de catégories « modernes » et pourvoyeur d'entités symétriques revenues de loin. Pour lui, s'évanouit jusqu'à l'opposition entre le sujet et l'objet $(1999,2001)$. Dans cet esprit, va-t-on se retrouver devant un monde devenu tout comme cette toile monochrome, uniformément pourpre, que son auteur, Alphonse Allais, se flattait d'avoir peinte ${ }^{30}$ ? N'ayons crainte. Latour voudrait saisir dans leur diversité les associations entre humains et non-humains (les "collectifs »), d'où l'éclairage de reliefs inédits. (Mais un parler neuf, au moins en apparence, ne produit pas toujours la vérité opératoire de ce qu'il voudrait faire accroire; $c f$. Jamard 1992b) Entre ces deux extrêmes, nos catégories de « Modernes » et l'ontologie latourienne (un troisième axe mène à la naturalisation des sciences humaines), Warnier, tout comme le MàP, ne choisit pas, malgré certaines tentations, car il entend résider ailleurs. Est-ce une option délibéré ? Est-ce la bonne? Nous verrons bien. Mais lorsque, pressé peutêtre de faire de monisme vertu, il embrasse, d'un seul mouvement « subjectivant ", des faits afférents à la paléo-anthropologie, à l'ethnologie du lointain, à la sociologie du présent, le risque est en dernier lieu, nous l'avons vu, celui d'un discours éclaté : pluraliste. Comment peut-il s'en prémunir? Comment atteindre au monisme tous terrains, contourner de prétendus technicismes? Eh bien, sotto voce, me vient cette adresse : «Allons, amis du MàP, encore un effort pour être vraiment latouriens ! ".

Il m'arrive d'exagérer : désir de se faire comprendre d'un seul trait. De plus, je me suis permis quelques diagnostics, jugements ou formules à l'emporte-pièce, car c'est le très proche que l'on doit connaître le mieux mais qui peut accepter la discussion sans arrière-pensées, enfin je l'espère. D'ailleurs, il est des critiques tacitement plus élogieuses que bien des dithyrambes proclamés; dans le cas présent, elles démontrent en tout cas que j'ai appliqué toute mon attention à une lecture captivante. Nous n'avons pas tous les mêmes idées (penser comme les autres, c'est ne pas penser), mais 
"Anthropologie » est un nom de famille, pas un nom propre. Nous ne pouvons que gagner, en restant dans nos maisonnées respectives, à des échanges et des empiétements réciproques. Somme toute, pour dire le sentiment d'un frontalier, le programme de recherche défini par le MàP dans un champ qui reste heureusement à défricher, apparaît bien assez généreux -et ambitieux !-, les observations attenantes sont bien assez riches, pour se passer des cautions philosophiques qui parfois brouillent les cartes ou voilent les enjeux. Les idées vaudront par leur progéniture, non par leurs généalogies. Des racines, point trop n'en faut: elles empêchent de bouger. Mais quoi qu'en pensent leurs divers lecteurs, et ce n'est pas là maigre mérite, les écrits de JeanPierre Warnier comme ceux du groupe « Matière à Penser » donnent sans aucun doute matières à réflexion.

\section{BIBLIOGRAPHIE}

Akrich, Madeleine (ed.)

1995 « Les objets de la médecine » (livraison thématique), Techniques \& culture 25-26.

Appadurai, Arjun

2001 Après le colonialisme. Les conséquences culturelles de la globalisation. Paris : Payot (préface de Marc Abélès). [Éd. orig. Modernity at Large. Cultural Dimensions of Globalization, University of Minnesota Press, 1996.]

Bédoucha, Geneviève

1987 «L'eau, amie du puissant » [...]. Une communauté oasienne du Sud tunisien. Paris : Éditions des Archives contemporaines («Ordres sociaux »).

Boas, Franz

1927 Primitive Art. New York : Dover Publications.

Boëtsch, Gilles \& Dominique Chevé (eds)

2000 Le Corps dans tous ses états. Regards anthropologiques. Paris : CNRS Éditions ( CNRS

anthropologie »).

Bonniol, Jean-Luc

1992 La Couleur comme maléfice. Une illustration créole de la généalogie des Blancs et des Noirs. Paris : Albin Michel (« Bibliothèque de synthèse »).

Boyer, Pascal

1997 La Religion comme phénomène naturel. Paris : Bayard Éditions (« Sciences »). [Éd. orig. The Naturalness of Religious Ideas, University of California Press, 1994.]

2001 Et l'homme créa les dieux. Comment expliquer la religion. Paris : Robert Laffont.

Calvin, William $\mathrm{H}$.

1994 « La naissance de l'intelligence », Pour la science 206 : 110-118.

Changeux, Jean-Pierre

2002 L’Homme de vérité. Paris : Paris : Éditions Odile Jacob (« Sciences »). 
Changeux, Jean-Pierre \& Alain Connes

1989 Matière à pensée. Paris : Éditions Odile Jacob.

Cosmides, Leda, John Tooby \& Jerome H. Barkow (eds)

1992 The Adapted Mind. Evolutionary Psychology and the Generation of Culture. New York : Oxford

University Press.

Cresswell, Robert

1994 « La nature cyclique des relations entre le technique et le social. Approche technologique de la chaîne opératoire ", pp. 275-289, in B. Latour \& P. Lemonnier (eds), De la préhistoire aux missiles balistiques. L'intelligence sociale des techniques. Paris : La Découverte («Recherches »).

1996 Prométhée ou Pandore ? Propos de technologie culturelle. Paris : Kimé (« Anthropologies »).

Cresswell, Robert \& François Sigaut (eds)

1987 « Des idées pour observer », Techniques \& culture 9 (livraison thématique).

Cresswell, Robert, Jean-Luc Jamard \& François Sigaut (eds)

1994 «Cultures de bêtes... Outils qui pensent? » (livraison thématique), Techniques \& culture 23-24.

Descola, Philippe

1994 « Pourquoi les Indiens d'Amazonie n'ont-ils pas domestiqué le pécari ? Généalogie des objets et anthropologie de l'objectivation », pp. 329-344, in B. Latour \& P. Lemonnier (eds), De la préhistoire aux missiles balistiques. L'intelligence sociale des techniques. Paris : La Découverte (« Recherches »).

Dessalles, Jean-Louis

2000 Aux origines du langage. Une histoire naturelle de la parole. Paris : Hermès Science Publications.

Dosse, François

1997 L'Empire du sens. L'humanisation des sciences humaines. Paris : La Découverte (« Poche-

Découverte ») (2ème édition).

Eisenstein, Elizabeth L.

1991 La Révolution de l'imprimé dans l'Europe des premiers temps modernes. Paris : La Découverte

(« Textes à l'appui-Anthropologie des sciences et des techniques »). [Éd. orig. The Printing

Revolution in Early Modern Europe, Cambridge (Mass.), Cambridge University Press, 1983, version abrégée et illustrée de The Printing Press as an Agent of Change, même éd., 2 tomes, 1979.]

Erikson, Erik Homburger

1982 Enfance et société. Neuchatel : Delachaux et Niestlé (1ère édition 1966).

Fine, Ben \& Ellen Leopold (eds)

1993 The World of Consumption. London : Routledge.

Foley, Robert

1995 Humans before Humanity. Cambridge : Cambridge University Press.

Foucault, Michel

2001 Foucault. Dits et écrits (2 vol. : I. 1954-1975; II. 1976-1988; éd. établie sous la dir. de Daniel Defert et François Ewald, avec la collab. de Jacques Lagrange). Paris : Gallimard (« Quarto »).

Fróis, Luís

1993 Traité de Luís Fróis, s. j. (1585) sur les contradictions de mœurs entre Européens \& Japonais, traduit du portugais par Xavier de Castro \& Robert Schrimpf et présenté par José Manuel García. Paris : Éd. Chandeigne-Librairie portugaise (« Magellane »). [1ère édition du texte de L. Fróis : 1585.] 
Gandoulou, Justin-Daniel

$1989 \mathrm{Au}$ cœur de la sape. Mœurs et aventures des Congolais à Paris. Paris : L'Harmattan.

Garrigues-Cresswell, Martine \& Béatrice Lecestre-Rollier

2001 « Gérer les aléas. Les sociétés du Haut Atlas marocain », Techniques \& culture 38 : 69-95.

Gelbert, Agnès

1997 « De l'élaboration au tour au tournage sur motte : difficultés motrices et conceptuelles »,

Techniques \& culture $30: 1-23$.

Geistdoerfer, Aliette

1999 « Aimer le mer : une technique du corps et de l'esprit », pp. 153-172, in J.-L. Jamard, A.

Montigny \& F.-R. Picon (eds), Dans le sillage des techniques. Hommage à Robert Cresswell. Paris :

L'Harmattan.

Goody, Jack

1979 La Raison graphique. La domestication de la pensée sauvage. Paris : Éditions de Minuit (« Le sens commun »). [Éd. orig. The Domestication of the Savage Mind, Cambridge, Cambridge University

Press, 1977.]

Guille-Escuret, Georges

1994 Le Décalage humain. Le fait social dans l'évolution. Paris : Kimé (« Anthropologies »).

Haudricourt, André-Georges

1987a « Relations entre gestes habituels, forme des vêtements et manière de porter les charges ", (1ère édition 1948), pp. 171-182, in

A.-G. Haudricourt, La Technologie science humaine. Recherches d'histoire et d'ethnologie des techniques. Paris : Éditions de la Maison des sciences de l'homme (préface de François Sigaut).

1987 « "La technologie, science humaine » (1ère édition 1964), pp. 37-46, in A.-G. Haudricourt, La Technologie science humaine. Recherches d'histoire et d'ethnologie des techniques. Paris : Éditions de la Maison des sciences de l'homme (préface de François Sigaut).

1987c « Nature et culture dans la civilisation de l'igname : l'origine des clones et des clans » (1ère édition 1964), pp. 298, in Haudricourt, André-Georges, La Technologie science humaine. Recherches d'histoire et d'ethnologie des techniques. Paris : Éditions de la Maison des sciences de l'homme (préface de François Sigaut).

Ivanoff, Jacques

1991 « Des ignames au riz. La dialectique du nomade et du sédentaire chez les Moken », Études rurales $120: 71-88$.

Jamard, Jean-Luc

1992a "Consomption d'esclaves et production de "races" : l'expérience caraïbéenne ",

L'Homme 122-123-124 : 209-234.

$1992 \mathrm{~b}$ « Faut-il être résolument amoderne ? Les sciences, les techniques, Bruno Latour et

l'anthropologie », Techniques \& culture 19 : 161-190.

1994 « Trop humains ? L'anthropomorphisme méthodologique : éthologie animale,

"IA"... anthropologie ", Techniques \& culture 23-24 : 159-218.

2000 « Des humeurs, des couleurs... et d'autres opérateurs », pp. 17-35, in J.-L. Jamard, E. Terray \& M. Xanthakou (eds), En substances. Textes pour Françoise Héritier. Paris : Fayard.

Jeannerod, Marc

2002 La Nature de l'esprit. Paris : Éditions Odile Jacob (« Sciences »). 
Julien, Marie-Pierre

1994 « Meubles chinois Made in France », pp. 79-98, in J.-P. Warnier (ed.), Le Paradoxe de la marchandise authentique. Imaginaire et consommation de masse. Paris : L'Harmattan.

Kaufmann, Jean-Claude

1997 Le Cour à l'ouvrage. Théorie de l'action ménagère. Paris : Nathan (« Essais \& Recherches »).

2001 Ego. Pour une sociologie de l'individu. Paris : Nathan (« Essais \& Recherches »).

Kawada, Junzo

1990 « Techniques du corps dans la technologie traditionnelle », pp. 111-140, in Kawada, Junzo (ed.), Boucle du Niger. Approches multidisciplinaires (vol. 2). Tokyo : Institut de recherche sur les langues et cultures d'Asie et d'Afrique, Tokyo University of Foreign Studies (en français). 1996 « Postures de portage et de travaux manuels - en rapport avec d'autres domaines de la vie japonaise », communication au colloque « Culture et usages du corps », Saint-Germain-en-Laye, 1er-4 mars 1996 (multicopié).

La Cecla, Franco

2002 Le Malentendu. Paris : Balland (« Voix et regards »; préface de Marc Augé). [Éd. orig. Il Malintenso, Bologna, Gius. Laterza \& Figli, 1997.]

Latour, Bruno

1991 Nous n'avons jamais été modernes. Essai d'anthropologie symétrique. Paris : La Découverte (« Armillaire »).

1992 Aramis, ou l'amour des techniques. Paris : La Découverte (« Textes à l'appui-Anthropologie des sciences et des techniques »).

1999 Politiques de la nature. Comment faire entrer les sciences en démocratie. Paris : La Découverte (« Armillaire »).

2001 L'espoir de Pandore. Pour une version réaliste de l'activité scientifique. Paris : La Découverte («Armillaire »). [Ed. orig. Pandora's Hope. Essays on the Reality of Sciences Studies, Cambridge (Mass.), Harvard University Press, 1999.]

Latour, Bruno \& Pierre Lemonnier

1994 « Introduction. Genèse sociale des techniques, genèse technique des humains ", pp. 9-24, in B. Latour \& P. Lemonnier (eds), De la préhistoire aux missiles balistiques. L'intelligence sociale des techniques. Paris : La Découverte (« Recherches»).

Leroi-Gourhan, André

1964 Technique et langage, t. I de Le Geste et la parole. Paris : Albin Michel (« Sciences d'aujourd'hui »).

1965 La Mémoire et les rythmes, t. II de Le Geste et la parole. Paris : Albin Michel (« Sciences d'aujourd'hui »).

1983 Mécanique vivante. Le crâne des vertébrés du poisson à l’homme. Paris : Fayard (« Le temps des sciences »; publié à partir d'une thèse principale de doctorat ès sciences soutenue en 1954).

Lestel, Dominique

2001 Les Origines animales de la culture. Paris : Flammarion.

Mauss, Marcel

1974 « Conceptions qui ont précédé la notion de matière » (1ère édition 1939), pp. 161-166 in Mauss, Marcel, Euvres (3 vol.), vol. 2 : Représentations collectives et diversité des civilisations. Paris : Éditions de Minuit (présentation de Victor Karady).

1985a « Rapports réels et pratiques de la psychologie et de la sociologie » (1ère édition 1924), pp. 283-330, in M.Mauss, Sociologie et anthropologie. Paris : Presses universitaires de France 
(«Quadrige »; présentation de Claude Lévi-Strauss).

1985 b « Les techniques du corps » (1ère édition 1936), pp. 365-386, in M. Mauss, Sociologie et anthropologie. Paris : Presses universitaires de France («Quadrige »; présentation de Claude LéviStrauss).

Merleau-Ponty, Maurice

1997 Phénoménologie de la perception. Paris : Gallimard (« Tel »). (1ère édition 1945)

Miller, Daniel E.

1987 Material Culture and Mass Consumption. Oxford/New York : Basil Blackwell (« Social

Achaeology Serie »).

Olivier de Sardan, Jean-Pierre

1996 « La violence faite aux données. De quelques figures de la surinterprétation en anthropologie », Enquête. Anthropologie, histoire, sociologie 3 : 31-59.

Parlebas, Pierre

1981 Lexique commenté en science de l'action motrice. Paris : INSEP, ministère de la Jeunesse, des sports et des loisirs.

1986 Éléments de sociologie du sport. Paris : Presses universitaires de France (« Sociologies »; préface de Raymond Boudon).

Picon, François-René

1999 « Le cheval dans le Nouveau Monde. Histoires culturelles », Études rurales 151-152: 51-75.

Schilder, Paul

1980 L'Image du corps. Paris : Gallimard (" Tel »). [Éd. orig. The Image and Appearance of the Human

Body, 1935, republ. : London, Routledge, 1999 (« International Library of Psychology »).]

Schlanger, Nathan

1991 « Le fait technique total. La raison pratique et les raisons de la pratique dans l'œuvre de Marcel Mauss », Terrain. Carnets du patrimoine ethnologique 16 : 114-130.

1994 « Piaget et Leroi-Gourhan. Deux conceptions biologiques des connaissances et des techniques », pp. 165-186, in B. Latour \& P. Lemonnier (eds), De la préhistoire aux missiles balistiques. L'intelligence sociale des techniques. Paris : La Découverte (« Recherches»).

Selz, Marion

1994 « L'ordinateur pense... Ou fait penser? », Techniques \& culture 23-24 : 351-370.

Shimekawa (auteur fictif)

1997 Manuel de l'oreiller (traduit du japonais ancien et présenté par Jean Cholley, introduction de Sugiura Saichirô). Paris : Éd. Philippe Piquier. [Éd. orig. Onna shimekawa oeshi-fumi, détournement d'un titre célèbre, avec doubles sens; litter. « Manuel de bandaison [par Shimekawa] pour posséder les femmes ", s.l., s.d.]

Sigaut, François

1987 «Préface », pp. 9-34, in Haudricourt, André-Georges, La Technologie science humaine.

Recherches d'histoire et d'ethnologie des techniques. Paris : Éditions de la Maison des sciences de l'homme.

1990 « De la technologie à l'évolutionnisme. L'œuvre de Pitt Rivers (1827-1900) », Gradhiva. Revue d'histoire et d'archives de l'anthropologie $8: 20-37$.

1991 « Un couteau ne sert pas à couper, mais en coupant. Structure, fonctionnement et fonction dans l'analyse des objets ", pp. 21-34, in Vingt-cinq ans d'études technologiques en préhistoire. Bilan et perspectives (introduction de Catherine Perlès; actes des 11èmes Rencontres internationales d'archéologie et d'histoires d'Antibes, 18-20/10/1990). Juan-les-Pin : édité par l'APDCA. 
Sperber, Dan

1996 La Contagion des idées. Théorie naturaliste de la culture. Paris : Éditions Odile Jacob.

Terradas, Ignasi

1988 Mal natural, mal social. Introducción a la teoría de las ciencias humanas. Barcelona : Barcanova.

Thomson, Michael

1979 Rubbish Theory. The Production and Destruction of Value. Oxford : Oxford University Press.

Van Gulik, Robert H.

1997 La vie sexuelle dans la Chine ancienne (traduit de l'anglais et du latin par Louis Évrard). Paris : Gallimard (« Tel »). [Éd. orig. Sexual Life in Ancient China. A Preliminary Survey of Chinese sex and society from ca. 1500 B.C. till 1644 A.D., Leiden, E. J. Brill, 1961.]

Warnier, Jean-Pierre (ed.)

1994 Le Paradoxe de la marchandise authentique. Imaginaire et consommation de masse. Paris :

L'Harmattan.

Washburn, Sherwood L.

1965 « An ape's eye view of evolution », in P. L. DeVore (ed.), The Origin of Man (symposium transcript). New York: Wenner-Gren Foundation for Anthropological Research.

Willems, Dick

1995 « Mesurer sa respiration : reconstituer le corps avec un objet médical »,

Techniques \& culture 25-26:55-70.

\section{NOTES}

*. En el original francés un segundo sentido de la primera parte del título sería

«Entrando en materia »

1. CC: Warnier, Jean-Pierre, Construire la culture matérielle. L'homme qui pensait avec ses doigts. Paris : Presses universitaires de France, 1999 (« Sciences sociales et sociétés »), 168 p. + biblio. 8 p.

2. «Selon Halbwachs, l'homme est un animal qui pense avec ses doigts» (Mauss 1974 : 162).

3. AM : Julien, Marie-Pierre \& Jean-Pierre Warnier (eds), Approches de la culture matérielle. Corps à corps avec l'objet. Paris : L'Harmattan, 1999 (« Connaissance des hommes »), 144 p., huit contributions (outre l'introduction de M.-P. Julien et la conclusion de J.-P. Warnier). Dommage que ce livre-là souffre grandement des carences techniques dont son éditeur est coutumier : nombreuses fautes et coquilles, fragilité de la brochure...

4. Je rappelle la « découverte », en 1911, de l'homme de Piltdown, le « chaînon manquant » à la mode de l'époque (crâne humain, mandibule simiesque), une supercherie qui ne sera dénoncée qu'en 1954 -alors que trois décennies s'étaient écoulées depuis l'exhumation de l'australopithèque bipède à petite tête-, ce délai de 43 ans témoignant de la force d'inertie du paradigme en question. (Dernière minute, l'hominidé Toumaï, 8 millions d'années BP, trouvé au Tchad : déjà surpassée, Lucy, 3 millions BP, cette jeunette est sur la touche.)

5. Warnier presse son lectorat de lui trouver une terminologie moins " jargonnante » que la sienne. Si elle l'est, peu importe, pourvu que l'on s'entende.

6. P. Parlebas, « Les tactiques du corps », AM : 29-43. 
7. Cf. des évocations infra, p. 224. Et pour les détails, surtout terminologiques, $c f$. Parlebas 1981; perspectives générales, analyses circonstanciées sur le système et la sociologie des sports dans Parlebas 1986. Sur un autre registre, l'importance du sport comme révélateur des problématiques identitaires (et de la construction de l'hexis corporel), voir les interprétations que propose Arjun Appadurai du cricket en Inde (2001, chap. IV).

8. C. Rosselin, «"Si tu vas un peu brusquement, tu te cognes contre l'armoire !" », AM : 107-117.

9. T. Putnam \& V. Swales, « Défaire et faire les habitudes dans le déménagement » (traduit par Warnier), AM : 119-134.

10. Dans Life of Brian, film du groupe « Monty Python ».

11. A. Jeanjean, "Travailler et penser une matière impensable », AM : 73-85.

12. « Nez à nez », entretien de F. Lecœur avec C. Rosselin, AM : 87-95.

13. F. Hoarau, « Trier, transporter à Emmaüs. Ethnographie, sens, et science de l'action ", $A M$ : 97-106.

14. À ce propos, $c f$. notamment un livre tout récent de M. Jeannerod (2002); strictement neurobiolologique, n'étaient quelques échappées : Changeux 2002 (chap.II et III en particulier).

15. N. Auray, «L'émergence du civisme dans une collectivité technique », AM : 55-71.

16. Nulle ironie dans cet emprunt : il serait malvenu de part d'en faire montre ici, puisque j'ai recouru çà et là, pour mon profit, au langage de la subjectivation lors d'une communication au colloque « MàP III » (dont la substance sera publiée sous peu); mais cela n'interdit pas d'en nuancer la portée, on le verra (cf. infra).

17. R. Pottier, « La place du corps dans les processus de socialisation : l'exemple du Confucianisme », AM : 45-54.

18. Jean-Luc Bonniol (1992) emploie l'expression, mais en un autre sens, assez voisin de la notion de « capital racial »-indexé, celui-ci, sur les phénotypes incorporant la trajectoire socio-historique de leurs porteurs-que j'applique à l'étude des dynamiques " coloristes » (notamment matrimoniales) liées aux rapports différentiels des hommes entre eux, et aux choses, dans les sociétés caraïbéennes héritières de l'esclavage colonial avec son « biracialisme » originel (pour un survol et des mises en perspective, cf. e.g. Jamard 1992a, 2000...).

19. Les Mélanésiens dont parle Haudricourt (1987c) ne proclament pas expressément que leurs clans sont comme des clones de tubercules, ni qu'eux-mêmes et leurs ancêtres sont comparables à des ignames. Les Yurok, Amérindiens de Californie, ne disent pas vraiment tout seuls que leur tube digestif enfin contrôlé dans l'espace du corps, c'est la rivière dont ils doivent maîtriser le cours (à la saison des pêches) et qui traverse le cercle de leur espace social (cf. Erikson 1982) : l'ethnologue les y aide un peu. Faut-il s'en étonner?

20. 2ème partie, «L'approvisionnement matériel moderne »: 81 pages (la 1ère partie, «Faire corps avec l'objet », en compte 64, mais 24 sont consacrées à une « société de la tradition », celle des Grassfields).

21. Comparez avec ce que filma Jean Rouch en Afrique, Les Maîtres fous, qui émut si fort nos aînés dans la carrière : « On doit pas montrer cela hors de nos murs! "; avec, aussi, une modalité distincte de construction « idéale » des substances sociales, le biais étant cette fois la stratégie de métissage : aux Antilles ou ailleurs, un type physique « proche » du « Blanc » permet l'hypergamie économique, mais le nanti peut s'unir à 
plus clair que soi pour se blanchir, par contiguïté ou dans sa progéniture (cf. supra, n. 18 , sur le « capital racial»).

22. Dans ces pages, l'auteur fait un juste sort - outre le post-modernisme en général - à Jean Baudrillard et à Michel Maffesoli, surtout au second : ses nombreux écrits ne forcent à mon sens ni la conviction ni le respect; ils seraient, eux, passibles me semblet-il de l'anathème lapidaire et un rien francophobe lâché par quelques critiques étatsuniens : Frog fog !, « Brouillard de mangeurs de grenouilles », mais dont ils frappent l'œuvre de Michel Foucault (il n'y a pas l'ombre d'une comparaison possible), sûrement pour l'avoir mal lue.

23. L'authenticité se définit comme n'étant pas « ...marchandisable, [mais les] systèmes d'appro-visionnement [offrent] un compromis paradoxal sous la forme de produits "authentiques" et cependant achetables " (CC:150).

24. Craignant de me répéter, j'ai omis de mes commentaires le chapitre 1 de $C C$, comme l'« Introduction » (Julien) et la « Conclusion » (Warnier) d'AM. Indiquons seulement que ces auteurs y soulignent, en particulier, à quel point certain vocabulaire de Mauss -qui reste leur inspirateur- semble aujourd'hui vermoulu. Mais si ce n'était pas le cas 65 ans après, ou plus, il y aurait de quoi s'inquiéter! (Par ailleurs, dans le chapitre 2 de CC, figure un développement sur les rapports entre les « choix » de la chasse et l'émergence des pratiques de réciprocité, trop connus des anthropologues de tous bords pour devoir les évoquer ici.)

25. Exemple : «Quand nous marchons, nous soulevons nos vêtements par devant pour ne pas les salir; les Japonais le font tellement haut par derrière qu'on leur voit tout le nord découvert » (Fróis 1993, chap. « Des hommes, de leurs personnes et de leurs vêtements »; « [comparaison] n ${ }^{\circ} 31 »: 47$ ).

26. Cf. la fameuse expérience imaginaire du " cerveau dans un bocal », aporie qui n'est pas totalement obsolète : que pense-t-il, ce cerveau, et surtout, va-t-on lui greffer un corps, ou le greffer sur un corps?

27. La prochaine livraison thématique de Techniques \& culture, concertée de longue main, en fera son miel.

28. Techniques comme savoirs et savoir-faire objectivés ou objectivables dans la relation à la matière, même dans le « travail sur soi » :cf.e.g. Descola 1994 : 332; Jamard $1994: 177$.

29. En psycholinguistique évolutive, $c f$. Dessalles 2000 : la fonction langagière en bonne et due forme fut retenue, sur un fond multicausal, en raison des avantages sélectifs médiatisés- de la communication d'informations fiables au sein de coalitions d'ordre politique existant dans les sociétés que formaient nos très lointains ancêtres. 30. Intitulée « Récolte de tomates sur les bords de la Mer Rouge par une troupe de cardinaux apoplectiques ».

\section{RÉSUMÉS}

L'auteur enchâsse d'abord bon nombre de ses réflexions dans un commentaire très détaillé sur deux ouvrages. Ceux-ci traitent de la « culture matérielle », sous l'angle d'une « incorporation », 
par chaque sujet humain, de la dynamique des objets, approche originale des techniques du corps. Ils illustrent ainsi les problématiques du groupe de travail « Matière à Penser ». Sur cette lancée, l'article développe des positions personnelles, mais qui recoupent les champs de recherche comme les catégories conceptuelles où semblent se retrouver la plupart des chercheurs du groupe «Techniques et culture». De la confrontation de l'un et l'autre programmes de recherche, naissent, en fin de parcours, des discussions plus générales, exemples à l'appui, qui touchent, surtout, à ces questions : Le technique se distingue-t-il du reste de la culture? Comment? Et la «culture matérielle »? Qu'est-ce qu'un « sujet » face à l'« objet »? Quant aux rapports entre pareilles réalités, peut-on, doit-on en rendre compte par la voie de l'interprétation? Par celle d'un causalisme qui ne récuse pas la réduction bien tempérée ?...

\section{At the heart of the subject : are bodies objects?}

The author embed many o his remarks in a very detailed commentary on two books. These works deal with «material culture » from the angle of the «incorporation » (or « objectification ») by each human subject of the dynamics of the objects, which is an original approach to techniques of bodies in their context. This is in line with the problematics of the research team « Matter for thought ». With this start the article develops positions which are personal to the author, but which interest research fields, such as the conceptual categories used by most of the researchers in the laboratory "Techniques et culture ", from the confrontation of these research programs are eventually engendered more general discussions which bury up these questions, through examples : is technology to be apart from the rest of culture? what is a subject? and the object? As for the relations between such realities, can one, must one takes them into account through interpretation ? Through relations of causality which does not reject a "well tempered" reductionnism?

\section{En el corazón del sujeto : ¿ el cuerpo objetivado ?*}

Para empezar el autor engarza un cierto número de reflexiones propias, al calor del muy detallado comentario que realiza acerca de dos obras. Aquellas tratan acerca de la «cultura material ", vista desde el ángulo de que cada sujeto humano "incorpora » la dinámica de los objetos, perspectiva esta original de las técnicas del cuerpo. También ilustran las problemáticas del grupo de trabajo "Matière à Penser» («Materia para Pensar»). A partir de estos presupuestos el artículo desarrolla posiciones personales, que sin embargo se entrecruzan con los campos de investigación y las categorías conceptuales entre las que parecen coincidir la mayor parte de los investigadores del grupo «Técnicas y cultura ». De la confrontación entre ambos programas de investigación nacen, en fin, trayectorias, discusiones más generales, ejemplos a los que se recurre como argumentos a favor, y que atañen, sobre todo, a estas cuestiones: $i$ Se distingue la técnica del resto de la cultura ? ¿ Cómo ? Y la « cultura material », ¿ cómo se define? ¿Qué es un «sujeto" frente a su "objeto"? En cuanto a las relaciones que existen entre realidades semejantes : ¿ Se puede, se debe acaso dar cuenta de ellas por vía de interpretación, o tal vez recurriendo a un causalismo que no rechace la reducción mesurada?

\section{INDEX}

Mots-clés : épistémologie, démarcations du technique, incorporation (des dynamiques d'objets), Julien (M.-P.), sujet (phénoménologique), techniques du corps, Warnier (J.-P.) 
AUTEUR

JEAN-LUC JAMARD

CNRS, UMR 8098 « Techniques et culture », 27 rue Paul Bert, 94204 Ivry cedex (jamard@ivry.cnrs.fr). 